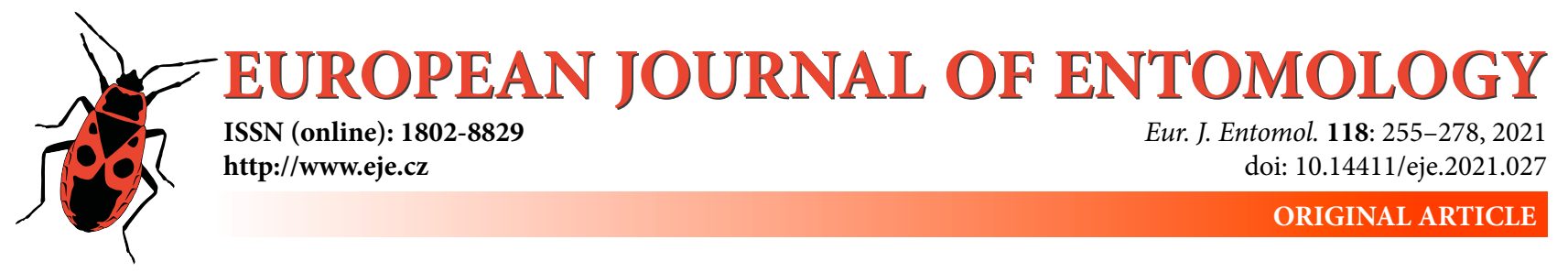

\title{
A dichotomous key and checklist for Mexican Athysanini leafhopper genera (Hemiptera: Cicadellidae) with a new species from the Oaxacan dry tropical forest
}

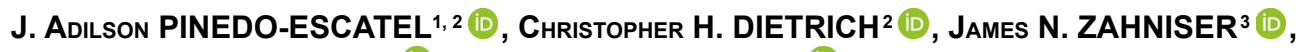 \\ Gustavo MOYA-RAYGOZA 4 (iD) and LIBERATO PORTILLO 4 (iD \\ ${ }^{1}$ Doctorado en Ciencias en Biosistemática, Ecología y Manejo de Recursos Naturales y Agrícolas (BEMARENA), Centro \\ Universitario de Ciencias Biológicas y Agropecuarias (CUCBA), Universidad de Guadalajara, Camino Ramón Padilla Sánchez \\ No. 2100, C.P. 45110, Las Agujas, Zapopan, Jalisco, México; e-mail: adilson1030@gmail.com \\ 2 Illinois Natural History Survey, Prairie Research Institute, University of Illinois, 1816 S. Oak St., Champaign, IL 61820, USA; \\ e-mail: chdietri@illinois.edu \\ ${ }^{3}$ USDA-APHIS-PPQ-NIS, National Museum of Natural History, Smithsonian Institution, P.O. Box 37012, Washington, DC $20013-$ \\ 7012, USA; e-mail: james.n.zahniser@usda.gov \\ ${ }^{4}$ Departamento de Botánica y Zoología, CUCBA, Universidad de Guadalajara, Camino Ramón Padilla Sánchez No. 2100, \\ C.P. 45110, Las Agujas, Zapopan, Jalisco, México; e-mails: moyaraygoza@gmail.com, liberatoportillo@gmail.com
}

Key words. Hemiptera, Auchenorrhyncha, Cicadellidae, Athysanini, distribution, checklist, key, new species, Mexico

Abstract. Most Neotropical forest-dwelling leafhopper species are rare and exhibit limited distributions. The Mexican leafhopper fauna is known to be highly diverse and identification of genera and species is difficult because no attempts have been made to provide comprehensive identification tools for the fauna. Here, a dichotomous key to all genera recognized within Mexico of the diverse but little studied leafhopper tribe Athysanini is provided. Spinulana josefinae Pinedo-Escatel sp. $\mathrm{n}$. is described and illustrated based on specimens collected in the dry tropical forest of Oaxacan mountains. A total of 46 genera and 146 species are now recognized in the checklist of Athysanini of Mexico. Notes on type repositories, distributional data and maps, and selected references are provided.

ZooBank Article Registration: http://zoobank.org/urn:Isid:zoobank.org:pub:FE90D7C2-C3FE-47B8-928F-68A0405C4141

\section{INTRODUCTION}

One of the largest herbivorous insect families worldwide is Cicadellidae (Hemiptera: Auchenorrhyncha: Cicadomorpha: Cicadoidea), which currently comprises more than 22,800 and in Deltocephalinae up to 7,100 species (Dietrich, 2005; Zahniser \& Dietrich, 2013). The Americas harbor a diverse leafhopper fauna comprising $22 \mathrm{sub}$ families (Dietrich, 2005). Within this family, the subfamily Deltocephalinae is the largest, with over 6800 species distributed in all major geographical regions. This morphologically diverse subfamily comprises 39 tribes, of which the forest-dwelling tribe Athysanini is the largest and most widespread with nearly 230 genera and 1,150 species known (Zahniser \& Dietrich, 2013).

Athysanini comprises 126 genera occurring in the New World with high diversity and richness in both northern and southern regions of the Americas (Linnavuori, 1959; Oman et al., 1990). This tribe, as presently defined, is polyphyletic, comprising all the genera that could not be placed into other, better-defined deltocephaline tribes (Zahniser \& Dietrich, 2013). The tribe includes many members that are highly specialized on host plants or habitats with distributional ranges limited and rarely extended beyond particular habitats or regions.

From the 1920 s to the 1940 s a comprehensive series of surveys on Mexican leafhoppers conducted by Dr. D.M. DeLong of Ohio State University (USA) and colleagues suggested that a large percentage of athysanine leafhopper species inhabit only specific areas within particular types of forests in western or central Mexico. Additional distributional records have not been published for most of the species documented in these original surveys. Thus, additional surveys are needed to further elucidate the distributions and conservation status of these endemic Mexican taxa.

Mexico is recognized as a megadiverse country with highly endemic genera and a mixture of Nearctic and Neotropical cicadellid faunas. The leafhopper fauna of Mexi- 
co has not been investigated thoroughly although a large number of short taxonomic contributions were published by DeLong based on specimens collected in the early to mid-1900s. Many regions remain understudied and knowledge of the fauna is far from complete. Also, identification of Mexican leafhoppers is difficult because there have been no attempts to provide comprehensive identification tools. Here we provide the first key to all genera of the tribe Athysanini known to occur in Mexico. A new species in the genus Spinulana DeLong, 1967 living on trees of the dry tropical forest from Oaxacan mountains is also described and the first annotated species checklist of this tribe for the country is included.

\section{MATERIALS AND METHODS}

\section{Morphological terminology}

Overall terminology herein follows Dietrich (2005), wing venation follows the system proposed by Anufriev \& Emeljanov (1988) and leg chaetotaxy follows Rakitov (1998). Nomenclatural changes and valid names followed Oman et al. (1990), Zanol (2008), Linnavuori (1959), and Oman (1949).

\section{Annotated list preparation}

Each checklist entry provides information in order as follows: taxon name, author and year of description, synonyms and original combination, citation, type material repository, distribution including Mexican political division, host plants, and additional relevant references at generic level. The genera and species are arranged alphabetically.

\section{Distributional data}

A series of layout maps were built following the criteria of Morrone et al. (2017) for Mexican biogeographic regionalization. Mapped points were generated and referenced using information from the literature, specimens collected by the first author and colleagues, and major museum specimen holdings (Table S1).

\section{Abbreviation of Mexican states used}

Baja California Sur (BCS); Campeche (CAMP); Ciudad de México (CDMX); Chihuahua (CHIH); Chiapas (CHIS); Coahuila (COAH); Estado de México (EDOMEX); Guerrero (GRO); Hidalgo (HGO); Jalisco (JAL); Michoacán (MIC); Morelos (MOR); Nuevo León (NL); Oaxaca (OAX); Puebla (PUE); Sinaloa (SIN); San Luis Potosí (SLP); Sonora (SON); Tabasco (TAB); Tamaulipas (TAMPS); Veracruz (VER); and Yucatán (YUC).

\section{Museum acronyms cited are as follows}

AMNH - American Museum of Natural History, New York, USA; CAJAPE - Colección de Auchenorrhyncha de J. Adilson Pinedo Escatel, Zapopan, Mexico; CAS - California Academy of Sciences, San Francisco, California, USA; CEAM - Colección de Insectos del Instituto de Fitosanidad, Colegio de Postgraduados, Estado de México, Mexico; CMNH - Carnegie Museum of Natural History, Pittsburgh, USA; CNIN - Colección Nacional de Insectos, Instituto de Biología, Universidad Nacional Autónoma de México, Ciudad de México, Mexico; CZUG - Centro de Estudios en Zoología, Centro Universitario de Ciencias Biológicas y Agropecuarias, Jalisco, Zapopan, Mexico; HNHM - Hungarian National Museum, Budapest, Hungary; INHS - Illinois Natural History Survey, Champaign, Illinois, USA; NHMUK - The Natural History Museum, London, UK; KUNHM - Kansas University Natural History Museum, Lawrence, USA; OSUC - Ohio State University, C.A. Triplehorn Insect Collection, Columbus, Ohio, USA; TAMU - Texas A\&M University, College Station, Texas,
USA; USNM - United States National Museum of Natural History, Washington, DC, USA.

\section{RESULTS}

A total of 46 genera and 146 species were formally recognized as occurring within the Mexican territory. Athysanini are reported from 22 states of Mexico, where Guerrero state is the richest with 32 genera and 70 species. Other states with high species richness are Michoacán, Jalisco, and Hidalgo. Most species occur over Sierra Madre del Sur province, Transmexican Volcanic Belt, and the Balsas Basin province which in part are in contact or over the Mexican Transition Zone. The genus with the largest number of species occurring in Mexico is Eutettix Van Duzee, 1892 followed by Mesamia Ball, 1907 and Ollarianus Ball, 1936 (Fig. 1), all of which also occur in the USA. Some other genera appear to be apparently exclusive (endemic) to Mexico and comprise species distributed in specific regions, e.g., Retusanus DeLong, 1945; Acunasus DeLong, 1945; Stoneana DeLong, 1943; etc. (DeLong, 1980), whereas many other taxa are monotypic and known from restricted locations (e.g., Pinedo-Escatel et al., 2016; Pinedo-Escatel \& Dietrich, 2020a). The distribution of Athysanini in Mexico is heterogeneous and some endemic genera vary in their apparent preferences for particular forest types such as Dry Tropical Forest, Pine/Oak Forest, and Montane Cloud Forest (e.g., Aguilar-Pérez et al., 2019).

\section{Tribe Athysanini Van Duzee, 1892 \\ Description}

Morphology. Length 2.5-7 mm. Body color green, brown, ochraceous, pale, reddish, orange or black, or combination, often including symmetrical stripes, spots or bands, or irregular patterns. Head subequal, wider or narrower than pronotum. Crown usually shagreen anteriorly with posterior part longitudinally striate or glabrous, anterior margin without distinct transverse carinae. Frontoclypeus weakly convex with texture shagreen. Anteclypeus parallel-sided or widening apically with apex following or slightly surpassing normal curve of gena. Lorum often wider than anteclypeus near base. Antennal ledge absent, antennal base near midheight of eyes. Pronotum with transverse striations very weak or absent, lateral margin usually much shorter than half eye width. Forewing macropterous with appendix narrow and restricted to anal margin; with 3 anteapical cells; with or without veinlets or false veins along costal margin. Hind wing venation fully developed, without pigmentation, RP-MA and MP-CuA separated by crossvein. Front femur AM row with only apical seta present; row AV usually with several short stout setae in basal half. Front tibia usually with dorsal macrosetal formula $1+4(\mathrm{AD}+\mathrm{PD})$. Mesotrochanter with one stout seta. Hind femur macrosetal formula $2+2+1$, without extra setae basad of usual set. Metatarsomere I not expanded apically, plantar setae simple, pecten usually with 3-4 platellae.

Genitalia. Male pygofer lobe with numerous macrosetae scattered over distal half; dorsal or ventral processes or teeth sometimes present; basolateral cleft present. Anal 


\section{Tribe Athysanini}

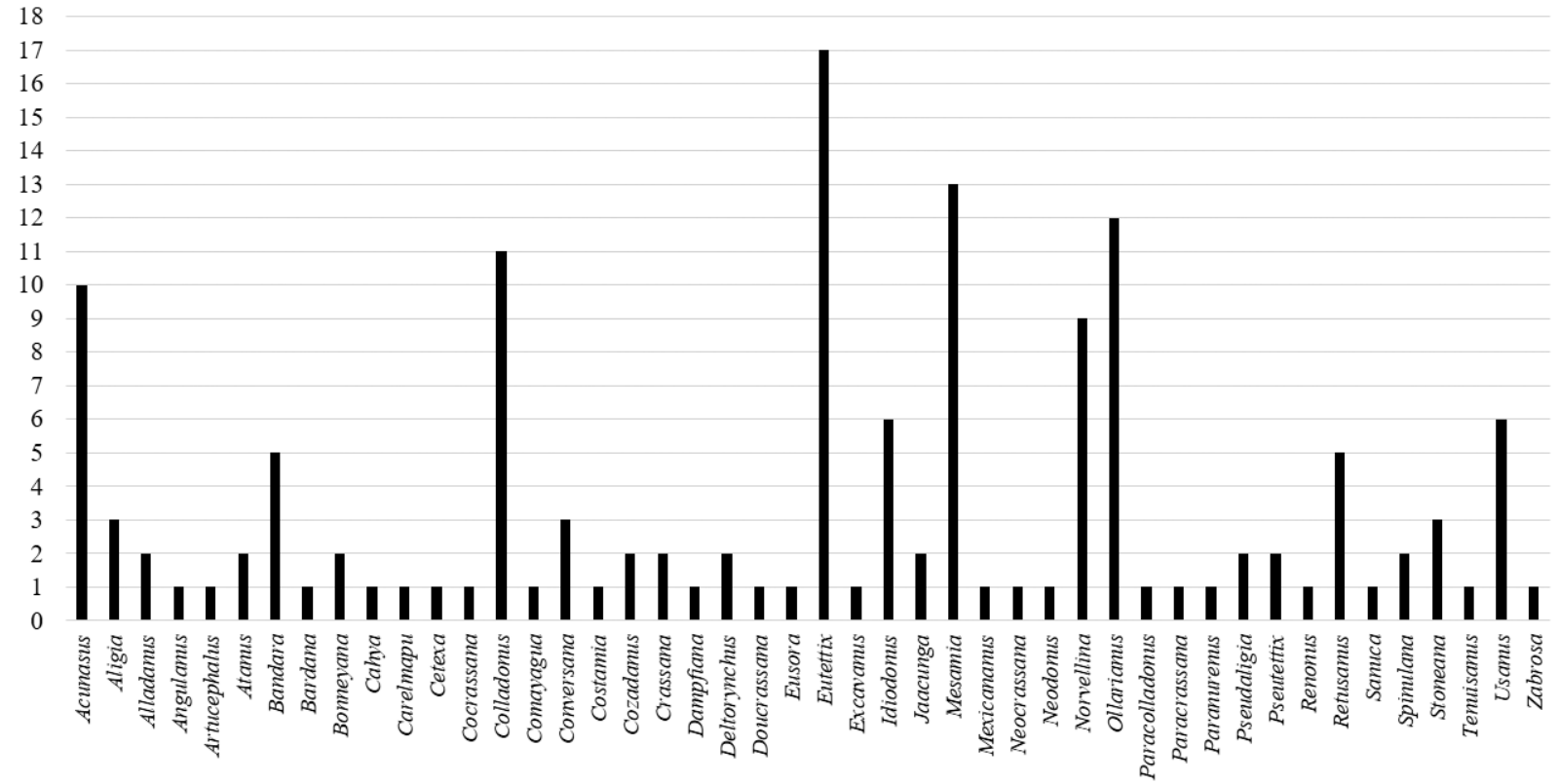

Fig. 1. Species numbers for each genus of the leafhopper tribe Athysanini in Mexico.

tube membranous or partially sclerotized but not elongate. Valve and subgenital plates free from each other, articulated with pygofer. Subgenital plate macrosetae uniseriate or somewhat irregularly arranged near lateral margin. Connective $\mathrm{Y}$ or H-shaped with anterior arms well separated and parallel to moderately divergent, stem movably articulated to aedeagus, without posterior extension or paraphysis. Style broadly bilobed basally; median anterior lobe not elongated. Aedeagus with or without processes. Phragma weakly sclerotized or membranous, dorsal connective absent.

Remarks. Most traits mentioned in the above tribal description may be found in one or more other tribes of Deltocephalinae. Previous phylogenetic analyses (e.g., Zahniser \& Dietrich, 2013) indicate that Athysanini is polyphyletic. The included genera have traditionally been retained here because they lack the distinctive, presumably derived, morphological traits diagnostic for other recognized deltocephaline tribes. Nevertheless, most Athysanini can be distinguished from other Deltocephalinae by a combination of the structure of the head (crown anterior margin without transverse carinae and anteclypeus parallel-sided or broadened distally) and male genitalia (anal tube short and incompletely sclerotized, connective Y-shaped and articulated to aedeagus, without posterior extension or paraphysis, dorsal connective absent).

\section{Key to genera of Mexican Athysanini (males)}

1 Head subequal or wider than pronotum (Fig. 9A) ................ 2

- Head distinctly narrower than pronotum (Fig. 9B) ............ 36

2 Dorsum marked with distinct bright orange bands (Figs 6G and $8 \mathrm{~F}$ )

- Dorsum without orange marks (Figs 8E, G and U)...

3 Head and pronotum marked with bright orange bands (Fig. $8 \mathrm{~F}$ ); pygofer without processes; aedeagus with median processes...
- Dorsum mostly matte orange with white spots and stripes (Fig. 6G); pygofer with processes; aedeagus without median processes. Bandara

4 Forewing with extra crossveins or veinlike pigment marks (Fig. 8C).

- Forewing without extra veins or veinlike pigment marks (Fig. $7 \mathrm{~T})$

5 Aedeagus without basal processes (Fig. 9C) ............................ 6

- Aedeagus with basal processes................................................ 8

6 Body with vermiculate color pattern or with numerous small tawny or brown pigment specks (Fig. 8O); style shorter than aedeagus and connective combined......................... Retusanus

- Body without vermiculate color pattern or numerous small pigment specks (Fig. 6B); style as long as or longer than aedeagus and connective combined ...

7 Pygofer processes arising caudally at or near posterior margin; processes less than one third pygofer length; apex of aedeagus dentate with or without minute processes

Pseudaligia

- Pygofer processes arising subapically from ventral margin and extended inside genital capsule, reaching posterior margin or not; processes longer than half pygofer length; apex of aedeagus smooth with or without small processes .........Aligia

8 Aedeagus with basal processes fused to shaft (Fig. 9D) ...... 9

- Aedeagus with basal processes articulated to shaft ............. 10

9 Crown with a black stripe or with a pair of triangular marks near anterior margin (Fig. 7P); crown surface concave; forewing with several crossveins and Pcu-A1 region only with two crossveins; pygofer with large and well sclerotized processes ...... ...Mesamia

- Crown usually with four spots on anterior margin; crown surface convex; forewing with few crossveins and Pcu-A1 region with more than 2 crossveins; pygofer with inconspicuous or small and not sclerotized processes ............ Norvellina

10 Corium and clavus with several extra crossveins (Fig. 7A); pygofer without appendages (Fig. 9E) ................... Costamia

- Corium and clavus without extra crossveins (Fig. 7F); pygofer with appendages (Fig. 9F) ............................................11 
11 Body color orange; costal area without or with 1-2 extra veins (Fig. 6O); aedeagus without or with small basal pro-

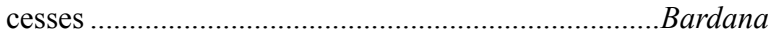

- Body color stramineous; costal area with more than 4 extra veins (Fig. 7F); aedeagus with long basal processes ... Eusora

12 Aedeagal atrium with lateral processes (Fig. 9G) .............. 13

- Aedeagal atrium simple (Fig. 9H) ...................................... 14

13 Crown without brown submarginal anterior band; pygofer lobe spine uniform from base to apex and not extended beyond dorsal margin (Fig. 6U) ..... Conversana

- Crown with brown submarginal anterior band; pygofer lobe spine broader at base than apex and extended beyond dorsal margin (Fig. 6D) ... Angulanus

14 Pygofer with processes or tooth (Fig. 9F and I) ................ 15

- Pygofer unmodified (Fig. 9E) ............................................. 27

15 Genital capsule with one pair of appendages (Fig. 9F) ...... 17

- Genital capsule with two pairs of appendages (Fig. 9I) ..... 16

16 Crown usually with four minute spots near anterior margin or if not then unicolorous (Fig. 7T); gonoduct not sclerotized...

Ollarianus

- Crown with brown transverse lines on median half; gonoduct well sclerotized at base ..................................... Duocrassana

17 Aedeagal base with processes (Fig. 9J) .............................. 18

- Aedeagal base without processes (Fig. 9E) ......................... 19

18 Dorsum marked with 4 symmetrical pairs of black spots, 1 pair each on head, pronotum, mesonotum, and forewings; forewing translucent (Fig. 7C); pygofer with straight processes; base of aedeagus with processes shorter than half shaft length

Cocrassana

- Dorsum marked with 2 symmetrical pairs of black spots; forewing brownish (Fig. 8D); pygofer with strong sinuous processes; base of aedeagus with processes longer than half shaft length

..Pseutettix

19 Pygofer appendages extended inside capsule (Fig. 9K) ..... 20

- Pygofer appendages extended externally (Fig. 9I) .............. 21

20 Apex of style apophysis expanded and rounded; aedeagus with or without medial processes ...........................Cozadanus

- Apex of style apophysis conical and tapered to a point; aedeagus with apical processes............................................ Eutettix

21 Crown midlength equal to or less than distance between eye and midline; anterior margin rounded, if not then conical and subparallel to posterior margin; apex of aedeagus without or with one process

- Crown midlength more tween eye and midline; anterior margin produced (Fig. 9L); apex of aedeagus with two processes ....................Tenuisanus

22 Crown and pronotum strongly arched above eyes (Fig. 9M).

Zabrosa

- Crown and pronotum slightly convex but not arched above eyes (Fig. 9N)

23 Aedeagal gonoduct sclerotized basad of atrium

...Neocrassana

Aedeagal gonoduct not sclerotized basad of atrium ............ 24

24 Body color whitish, with ochraceous, brown, and black markings; crown with black paired spots and lines (Fig. 8Q); aedeagus without processes....................................... Spinulana

- Body color yellow, stramineous, blackish, brownish or rarely ochraceous; crown with black paired spots and yellow, black or brown transverse unpaired lines (Fig. 7U and S); aedeagus with processes (Fig. 9D and J)......................................... 25

25 Head much wider than pronotum; crown with anterior margin rounded; forewing translucent (Fig. 6T and Aa); aedeagus with subapical processes... Comayagua
- Head slightly wider than pronotum or subequal; crown with anterior margin pointed; forewing infused with yellow, brown or black (Fig. 7U and $\mathrm{Bb}$ ); aedeagus with apical processes ...

.. 26

26 Apical processes of aedeagus uniform, simple, and usually crossed ............................................................. Colladonus

- Apical processes of aedeagus sinuate, ornamented, and not crossed .........................................................Paracolladonus

27 Crown or anterior margin of head with one black pair of spots (Figs 70 and $8 \mathrm{~B}$ )

Crown or anterior margin of and $8 \mathrm{~A}$ )

28 Crown with complete or incomplete transverse black, white or brown bands or maculae; anterior margin of crown slightly produced

29

Crown only with black spots; anterior margin of crown rounded (Fig. 8R) ........................................................... 31

29 Base of aedeagus with paired or unpaired processes (Fig. 9J) Idiodonus

- Base of aedeagus simple, without processes (Fig. 9C) ...... 30

30 Aedeagus with paired apical processes .....................Jaacunga

- Aedeagus with a single process ............................Paranurenus

31 Aedeagal shaft with medial or apical processes and flanges developed. Usanus

- Aedeagal shaft simple, only with one long apical pair of processes, without flanges....................................... Bonneyana

32 Crown with a single transverse black band (Fig. 8A) ........ 33 Crown unicolorous (Fig. 7C)............................................ 34

33 Aedeagal shaft with lateral flanges; gonoduct not sclerotized basad of atrium Paracrassana

- Aedeagal shaft without lateral flanges; gonoduct well sclerotized basad of atrium ......................................... Crassana

34 Aedeagus with apical processes ....................................... 35 Aedeagus without apical processes .............................. Cetexa

35 Body yellowish (Fig. 6R); processes of aedeagus slender and simple, uniform.......................................................... Cahya

- Body brownish or blackish (Fig. 6F); processes of aedeagus broad and bifurcated, sinuous..................................... Atanus

36 Pygofer with appendages (Fig. 9F and I) .......................... 37

- Pygofer without appendages (Fig. 9E) ................................. 44

37 With two sets of pygofer appendages (Fig. 9I) ................... 38

- With one set of pygofer appendages (Fig. 9F)..................... 39

38 Head yellowish, pronotum reddish and wings brown; forewing venation simple (Fig. $8 \mathrm{P}$ ); aedeagus with minute subapical dorsal processes...............................................Sanuca

- Head, prontum and wings with orange marks; forewing with many crossveins (Fig. 8S); aedeagus without dorsal process.

39 Aedeagus with basal processes (Fig. 9J) ................. Alladanus Aedeagus without basal processes (Fig. 9C and $\mathrm{H}$ ) ........... 40

40 Pygofer appendage thin, arising caudally and strongly sinuous, shorter than posterior margin; aedeagus with a single process on dorsal surface, without flanges ............Dampfiana

- Pygofer appendage arising ventrally or from posterior margin usually beyond capsule, or if arising caudally and shorter than posterior margin then thick and straight; aedeagus without process and often with variable flanges ............................. 41

41 Pygofer appendage serrated mesad or apically................... 42

- Pygofer appendage somewhat corrugate or smooth with black

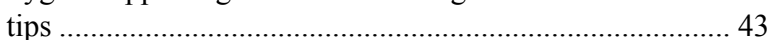

42 Body color yellowish with orange marks; anterior margin of crown produced (Fig. 7E); pygofer appendage serrated mesad. Deltorynchus 
- Body color entirely yellowish; anterior and posterior margin of crown subparallel (Fig. 7G); pygofer appendage serrated apically ............................................................... Excavanus

43 Crown with anterior margin produced, median length greater than length next to eyes (Fig. 6A); aedeagal preatrium well developed. Acunasus

- Crown with anterior and posterior margin subparallel, median length nearly equal to length next to eyes (Fig. 6E); aedeagal preatrium not developed ...................................Artucephalus

44 Body length above $7 \mathrm{~mm}$; fuscous with extra crossveins on corium (Fig. 7Q and X) .................................. Mexicananus

- Body length below $7 \mathrm{~mm}$; stramineous or yellow without extra crossveins.

45 Crown with a black band near anterior margin and distal area yellowish; apex of subgenital plate filamentous and extended beyond posterior margin of pygofer . Carelmapu

- Crown with a pair of black spots on anterior margin with minute red and black specks in distal area (Fig. 7R); apex of subgenital plate pointed and shorter than posterior margin of pygofer.. Neodonus

\section{Spinulana josefinae Pinedo-Escatel, sp. $\mathbf{n}$.}

Figs 2A-E, 3A-G

ZooBank taxon LSID:

4975B064-D888-4895-A00A-C4E0A15D0111

\section{Diagnosis}

Spinulana josefinae sp. n. can be distinguished by the combination of the following characters: head slightly produced, dorsal coloration light-brown with yellowish marks, crown with a large black spot beside each eye and four minute black spots adjacent to midline near anterior margin, and pygofer with bifid asymmetrical caudal process.

\section{Description}

External morphology. Overall color light-brown with dorsal, ventral and anterior yellowish marks (Fig. 2A-E). Crown yellowish with a light-brown broad line on anterior margin; two pairs of symmetrical black spots, outer pair next to eyes and larger than inner pair on midline; ecdysial line with arcuate light-brown band with two minute black spots centrally; midlength shorter than eye to midline (Fig. 2A-B). Ocellocular area tapering to ocelli; a black spot above antennal pit. Ocelli surrounded by triangular black spots. Frontoclypeus mostly yellowish with some transverse black lines arising mesad of lateral suture, upper area with pair of triangular black marks, lower area marked with black; midline yellowish. Antleclypeus with inverted black T-shaped macula mediall, yellowish laterally. Lorum mostly yellowish with margins black. Genae yellowish with a black marks below antenna (Fig. 2C). Pronotum with brownish and ivory transversal bands, a paired slender black stripes posteriorly. Forewing translucent. Veins dark-brown (Fig. 2D). Venter yellowish with some blackmarks. Legs with black and brown patches (Fig. 2 E).

Male genitalia. Pygofer lobe in lateral view longer than tall, squarish, macrosetae long and reduced with two or one row of four setae near posterior margin (Fig. 3A); pygofer process on ventral margin asymmetrically bifurcated in ventral view (Fig. 3B). Segment X one third as long as py- gofer; dorsal and lateral sides fully sclerotized; base to apex uniform and rectangular. Valve weakly projected posterad with rounded apical margin; $2.2 \times$ wider than long. Plate extended to pygofer apex; macrosetae uniseriate laterad, concentrated near midlength with fine setae intercalated; apex truncate (Fig. 3C). Style broad basally with preapical lobe weakly developed, apophysis very short, straight, not expanded, apex rounded and blunt (Fig. 3E). Connective stem apex slightly emarginated, shorter than arms (Fig. 3D). Aedeagus curved dorsad; without basal processes; shaft slender with lateral flanges; apex rounded with minute notch; gonopore apical and wide as shaft (Fig. 3F and G).

Female genitalia. Unknown.

Immature stages. Unknown.

Measurements. Body length, male 5.10-5.32 mm and female unknown; head width $1.64 \mathrm{~mm}$; crown length 0.25 $\mathrm{mm}$; crown width $0.81 \mathrm{~mm}$; eye length $0.21 \mathrm{~mm}$; eye width $0.60 \mathrm{~mm}$; width between ocelli $0.57 \mathrm{~mm}$; ocellocular area length $0.43 \mathrm{~mm}$; ocellocular area width $0.10 \mathrm{~mm}$; frontoclypeus length $0.95 \mathrm{~mm}$; frontoclypeus median width $0.66 \mathrm{~mm}$; frontoclypeus apex width $0.27 \mathrm{~mm}$; anteclypeus length $0.27 \mathrm{~mm}$; anteclypeus width $0.26 \mathrm{~mm}$; lorum length $0.32 \mathrm{~mm}$; lorum width $0.23 \mathrm{~mm}$; pronotum length 0.67 $\mathrm{mm}$; pronotum width $1.53 \mathrm{~mm}$; scutellum length $0.68 \mathrm{~mm}$; scutellum width $1.08 \mathrm{~mm}$; forewing length $4.0 \mathrm{~mm}$.

Etymology. The species is named in honor of the first author's mother, Josefina Escatel Sánchez.

Type material. Holotype $\hat{\delta}$ (INHS): MEXICO, Oaxaca, rt 190, km \#73, $3 \mathrm{~km}$ N San Pedro Totolapan, $1220 \mathrm{~m}, 16^{\circ} 43^{\prime} 5^{\prime \prime} \mathrm{N}$, 96¹9'29"W, 7.ii.2001, C.H. Dietrich Coll, sweeping 01-059-01. Paratype $\delta$ (CAJAPE): MEXICO, Oaxaca, Santa Maria Ecatepec, Carr. 190 Oaxaca-Tehuantepec, $8.2 \mathrm{~km}$-Este La Reforma, $16^{\circ} 24^{\prime} 24.3^{\prime \prime} \mathrm{N}, 95^{\circ} 42^{\prime} 35.9^{\prime \prime} \mathrm{W}, 536 \mathrm{~m}$ a.s.1., 26.vii.2019, PinedoEscatel, Brendan Morris y Juvenal Aragon Cols., Trampa de Luz, MEXOAX198.

Distribution. Mexico: Oaxaca [San Pedro Totolapan (holotype; type locality) and La Reforma (paratype)], Fig. 5 Q.

Habitat. Oaxacan dry tropical forest.

Remarks. This species differs from $S$. varigata DeLong and $S$. spinosa DeLong in having one short and asymmetrically bifid caudal pygofer process (Fig. 2A and B). The new species belongs to a distinct endemic group recently expanded based on study of DeLong's type material and other specimens (Pinedo-Escatel \& Dietrich, 2020a).

\section{Annotated checklist of Mexican Athysanini}

Order Hemiptera

Suborder Auchenorrhyncha

Infraorder Cicadomorpha

Superfamily Membracoidea

Family Cicadellidae

Subfamily Deltocephalinae

Tribe Athysanini

\section{Genus Acunasus DeLong, 1945}

Acunasus DeLong, 1945a: 199 (type species: Acunasus nigriviridis DeLong, 1945)

Mexican Transition Zone and Neotropical; distribution in Mexico Fig. 4A 


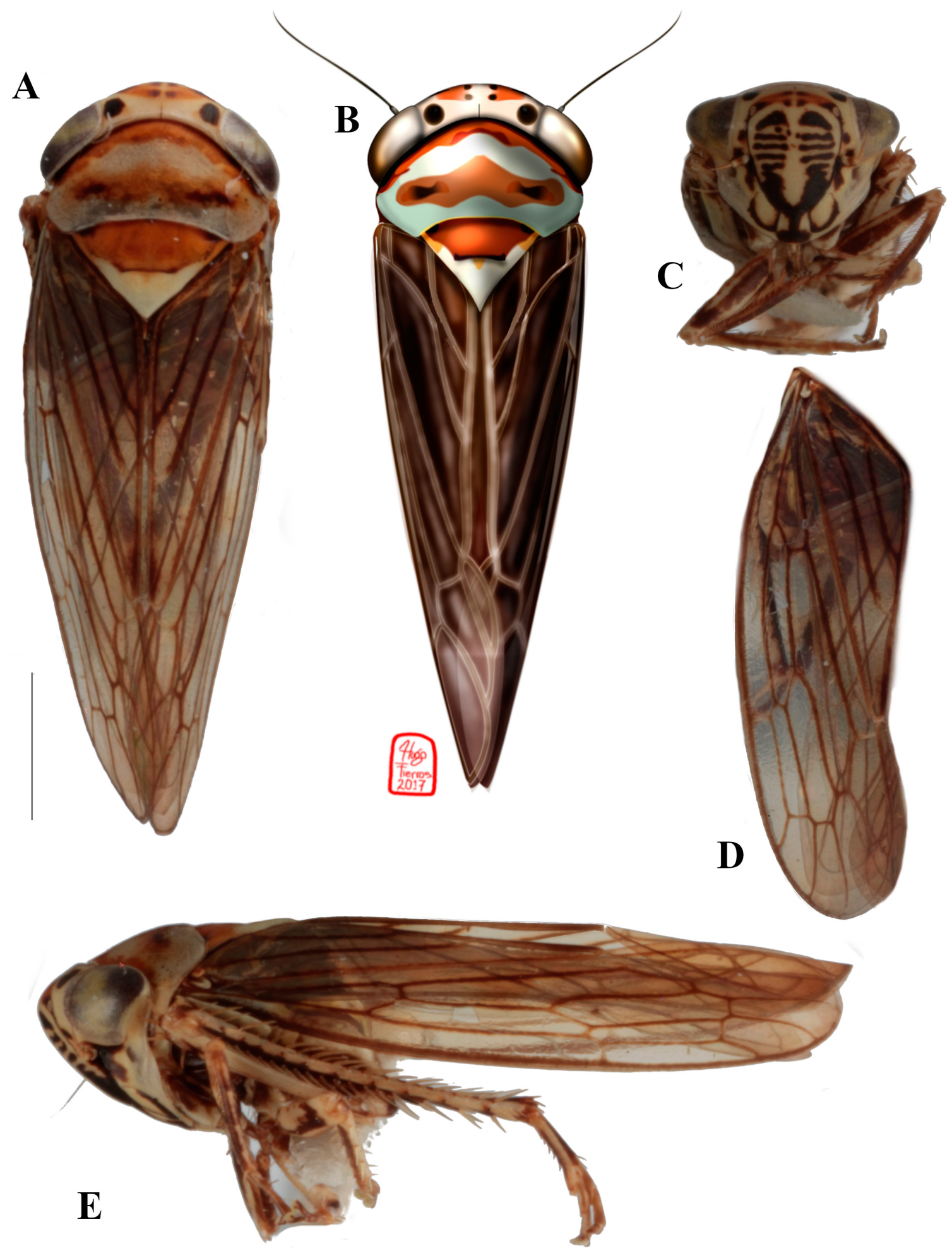

Fig. 2. Holotype of Spinulana josefinae sp. n. A-B - habitus, dorsal view; C - anterior view; D - forewing; E - lateral view. Scale bar $1 \mathrm{~mm}$.

\section{DeLong (1980)}

Host: Quercus spp.; Pinus spp.

Fig. $6 \mathrm{~A}$ and $\mathrm{H}$

\section{Acunasus angustatus DeLong, 1980}

Acunasus angustatus DeLong, 1980: 69

OSUC (holotype)

Endemic to Mexico (GRO)

\section{Acunasus brunneus DeLong, 1945}

Acunasus brunneus DeLong, 1945a: 200

OSUC (holotype); USNM (paratypes)

Endemic to Mexico (GRO)

Acunasus capitatus DeLong, 1945

Acunasus capitatus DeLong, 1945a: 204

OSUC (holotype and paratypes)

Endemic to Mexico (GRO; JAL) 


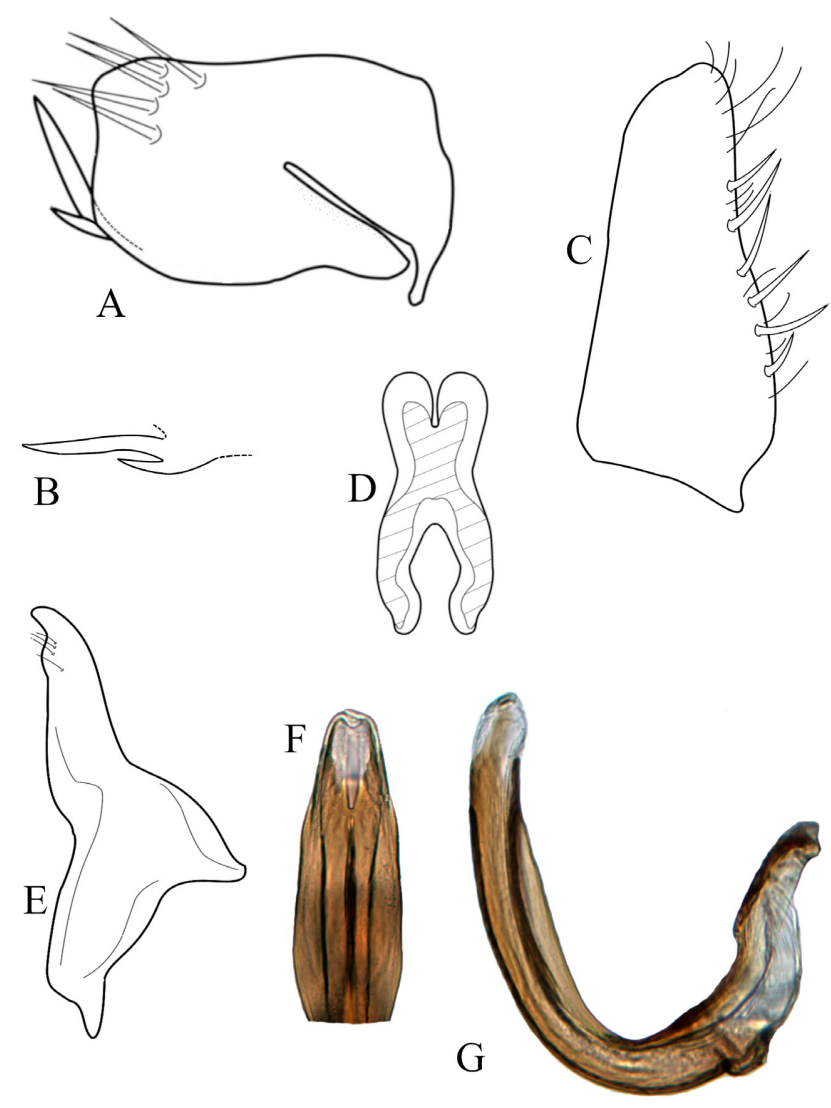

Fig. 3. Male genitalia of Spinulana josefinae sp. n. A - pygofer, lateral view; $\mathrm{B}$ - pygofer process, ventral view; $\mathrm{C}$ - subgenital plate, ventral view; $D$ - connective, ventral view; $E$ - right style, ventral view; $\mathrm{F}$ - aedeagus, posterior view; $\mathrm{G}$ - aedeagus, lateral view.

\section{Acunasus clavatus DeLong, 1945}

Acunasus clavatus DeLong, 1945a: 204

OSUC (holotype and paratypes)

Endemic to Mexico (GRO)

\section{Acunasus cruciatus DeLong, 1945}

Acunasus cruciatus DeLong, 1945a: 200

OSUC (holotype)

Endemic to Mexico (GRO)

\section{Acunasus hyalinus DeLong, 1945}

Acunasus hyalinus DeLong, 1945a: 202

OSUC (holotype); INHS and CNIN (other material)

Endemic to Mexico (GRO)

\section{Acunasus luteus DeLong, 1945}

Acunasus luteus DeLong, 1945a: 205

OSUC (holotype); OSUC and USNM (paratypes); CAJAPE (other material)

Endemic to Mexico (GRO; HGO)

\section{Acunasus nigriviridis DeLong, 1945}

Acunasus nigriviridis DeLong, 1945a: 199

OSUC (holotype and paratypes); CEAM, INHS, TAMU, OSUC, and CNIN (other material)

Endemic to Mexico (GRO)

\section{Acunasus venosus DeLong, 1945}

Acunasus venosus DeLong, 1945a: 199

OSUC (holotype)

Endemic to Mexico (GRO)

\section{Acunasus viridis DeLong, 1980}

Acunasus viridis DeLong, 1980: 69

OSUC (holotype and paratypes)

Endemic to Mexico (GRO; MIC)

\section{Genus Aligia Ball, 1907}

Eutettix (Aligia) Ball, 1907: 53 (type species: Jassus jucundus Uhler, 1877)

Nearctic and Mexican Transition Zone; distribution in Mexico Fig. 4B

Kramer \& DeLong (1968), Oman (1949), Hepner (1942a)

Fig. 6B and I

Aligia alvona Kramer \& DeLong, 1968

Aligia alvona Kramer \& DeLong, 1968: 169

OSUC (holotype); USNM (paratypes)

Endemic to Mexico (GRO; HGO; CDMX; VER)

Aligia bicolor Kramer \& DeLong, 1968

Aligia bicolor Kramer \& DeLong, 1968: 171

OSUC (holotype); USNM (paratypes)

Endemic to Mexico (CDMX)

\section{Aligia mexicana Kramer \& DeLong, 1968}

Aligia mexicana Kramer \& DeLong, 1968: 169

OSUC (holotype); USNM (paratypes)

Endemic to Mexico (CDMX; VER; HGO)

Genus Alladanus DeLong \& Harlan, 1968

Alladanus DeLong \& Harlan, 1968: 147 (type species: Alladanus cephalatus DeLong \& Harlan, 1968)

Mexican Transition Zone and Neotropical; distribution in Mexico Fig. 4C

Fig. 6C and J

\section{Alladanus cephalatus DeLong \& Harlan, 1968}

Alladanus cephalatus DeLong \& Harlan, 1968: 148

USNM (holotype)

Endemic to Mexico (VER)

Alladanus mexellus DeLong \& Harlan, 1968

Alladanus mexellus DeLong \& Harlan, 1968: 148

OSUC (holotype and paratypes)

Endemic to Mexico (MIC; VER; OAX)

Genus Angulanus DeLong, 1946

Idiodonus (Angulanus) DeLong, 1946a: 30 (type species: Idiodonus incisurus DeLong, 1946)

Mexican Transition Zone and Neotropical; distribution in Mexico Fig. 4D

DeLong (1983, 1984); Nielson (1988)

Fig. $6 \mathrm{D}$ and $\mathrm{K}$

\section{Angulanus incisurus (DeLong, 1946)}

Idiodonus (Angulanus) incisurus DeLong, 1946a: 30

Angulanus incisurus: Nielson, 1988: 133

OSUC (holotype and paratypes)

Endemic to Mexico (EDOMEX; MOR; JAL; OAX; GRO)

Genus Artucephalus DeLong, 1943

Artucephalus DeLong, 1943a: 654 (type species: Artucephalus fasciatus DeLong, 1943)

Neotropical; distribution in Mexico Fig. 4E

Fig. $6 \mathrm{E}$ and $\mathrm{L}$

Artucephalus fasciatus DeLong, 1943

Artucephalus fasciatus DeLong, 1943a: 654

OSUC (holotype and paratypes)

Endemic to Mexico (GRO) 


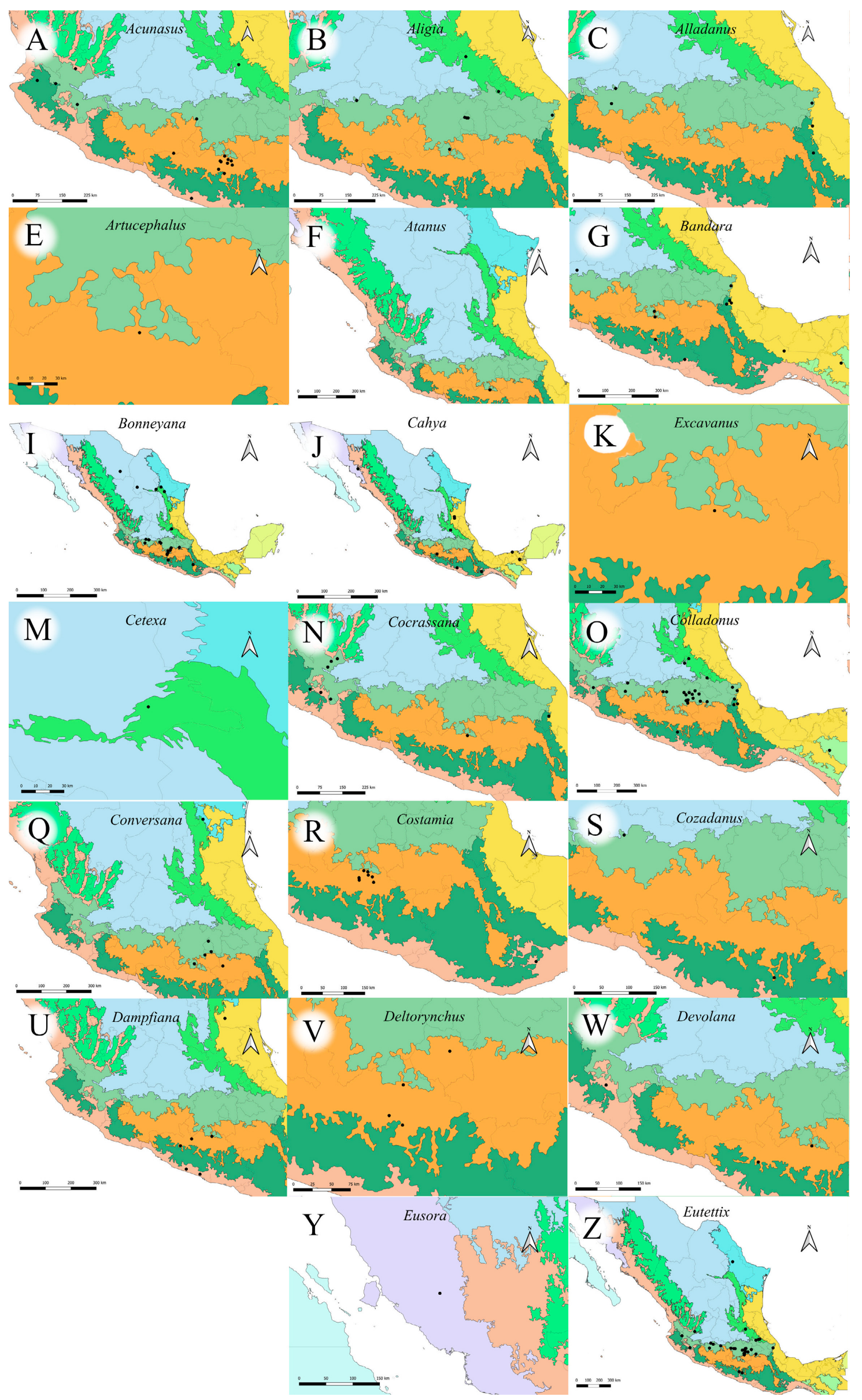

Fig. 4. Distributions of genera of Athysanini in Mexico. 


\section{Genus Atanus Oman, 1938}

Atanus Oman, 1938: 381 (type species: Eutettix dentatus Osborn, 1923)

Alanus DeLong \& Hershberger, 1947a: 231 (synonymized by Linnavuori, 1959: 296)

Nearctic and Neotropical; distribution in Mexico Fig. 4F

Beamer (1943), Oman (1949), DeLong (1978), Young (1957), Linnavuori (1959)

Fig. $6 \mathrm{~F}$ and $\mathrm{N}$

Atanus albidus (DeLong \& Hershberger, 1947)

Alanus [sic] albidus DeLong \& Hershberger, 1947: 231

USNM (holotype and paratypes)

Mexico (SIN) and Panama

Atanus mexicanus DeLong, 1978

Atanus mexicanus DeLong, 1978: 490

OSUC (holotype)

Endemic to Mexico (GRO)

Genus Bandara Ball, 1931

Bandara Ball, 1931: 93 (type species: Eutettix johnsoni Van Duzee, 1894)

Nearctic, Mexican Transition Zone, and Neotropical; distribution in Mexico Fig. 4G

DeLong (1980), Oman (1949), Knull (1946)

Fig. $6 \mathrm{G}$ and $\mathrm{N}$

Subgenus Bandara (Bandara) Ball, 1931

Bandara (Bandara) Ball, 1931: 93 (type species: Eutettix johnsoni Van Duzee, 1894)

Bandara (Bandara) lyrata DeLong, 1980

Bandara (Bandara) lyrata DeLong, 1980: 64

OSUC (holotype)

Endemic to Mexico (CHIS)

Bandara (Bandara) procera DeLong, 1980

Bandara (Bandara) procera DeLong, 1980: 64

OSUC (holotype and paratypes)

Endemic to Mexico (GRO)

Bandara (Bandara) similis DeLong, 1980

Bandara (Bandara) similis DeLong, 1980: 63

OSUC (holotype and paratypes)

Endemic to Mexico (GRO; OAX)

Bandara (Bandara) spinella DeLong, 1980

Bandara (Bandara) spinella DeLong, 1980: 63

OSUC (holotype and paratypes)

Endemic to Mexico (GRO; MIC)

Subgenus Bandara (Bandarana) DeLong, 1980

Bandara (Bandarana) DeLong, 1980: 64 (type species: Bandara mimica DeLong, 1980)

Bandara (Bandarana) mimica DeLong, 1980

Bandara (Bandarana) mimica DeLong, 1980: 64

OSUC (holotype and paratypes)

Mexico (VER) and Guatemala

\section{Genus Bardana DeLong, 1980}

Bardana DeLong, 1980: 65 (type species: Bardana depressa DeLong, 1980)

Mexican Transition Zone and Neotropical; distribution in Mexico

Fig. $4 \mathrm{H}$

Fig. $6 \mathrm{O}$ and $\mathrm{V}$

Bardana depressa DeLong, 1980

Bardana depressa DeLong, 1980: 65

OSUC (holotype and paratypes)

Endemic to Mexico (EDOMEX; MOR)

\section{Genus Bonneyana Oman, 1949}

Bonneyana Oman, 1949: 115 (type species: Thamnotettix schwar$z i$ Ball, 1911)

Nearctic, Mexican Transition Zone, and Neotropical; distribution in Mexico Fig. 4I

DeLong (1946a)

Host: Pinus spp.

Fig. $6 \mathrm{P}$ and $\mathrm{W}$

Bonneyana caldwelli (DeLong, 1946)

Idiodonus caldwelli DeLong, 1946a: 16

Bonneyana caldwelli: Nielson, 1988: 132

Idiodonus apertus DeLong, 1946a: 14

OSUC (holotype and paratypes); CAJAPE (other material)

Endemic to Mexico (GRO; MOR; MIC; PUE)

Bonneyana schwarzi (Ball, 1911)

Thamnotettix schwarzi Ball, 1911a:197

Idiodonus schwarzi: Ball, 1937: 27

Bonneyana schwarzi: Oman, 1949: 15

USNM (holotype); OSUC, INHS, and CAJAPE (other material)

Mexico (COAH; NL; CHIH) and USA

Genus Cahya Linnavuori, 1959

Cahya Linnavuori, 1959: 276 (type species: Thamnotettix pulchellus Osborn, 1923)

Mexican Transition Zone and Neotropical; distribution in Mexico Fig. 4J

DeLong (1945b)

Fig. 6Q and $\mathrm{X}$

Cahya variabilis (DeLong, 1945)

Chlorotettix variabilis DeLong, 1945b: 27

Cahya variabilis: Linnavuori, 1959: 278

OSUC (holotype and paratypes); CMNH, INHS, CAJAPE, and CAS (other material)

Mexico (TAB; GRO; SON; HGO; SLP; OAX) and Guatemala

Genus Carelmapu Linnavuori, 1959

Carelmapu Linnavuori, 1959: 220 (type species: Carelmapu scutellaris Linnavuori, 1959)

Neotropical; distribution in Mexico Fig. 4L

Zanol (1989)

Fig. $8 Y$

Subgenus Carelmapu (Carelmapu) Linnavuori, 1959

Carelmapu Linnavuori, 1959: 220 (type species: Carelmapu scutellaris Linnavuori, 1959)

Carelmapu (Carelmapu) mexicanus Zanol, 1989

Carelmapu (Carelmapu) mexicanus Zanol, 1989: 368

NHMUK (holotype)

Endemic to Mexico (GRO)

Genus Cetexa Oman, 1949

Cetexa Oman, 1949: 129 (type species: Thamnotettix graecula Ball, 1901)

Nearctic and Mexican Transition Zone; distribution in Mexico Fig. 4M

Fig. 6R and $\mathrm{Y}$

\section{Cetexa graecula (Ball, 1901)}

Thamnotettix graecula Ball, 1901: 6

Thamnotettix graeculus: Van Duzee, 1916: 74

Doleranus graeculus: DeLong \& Caldwell, 1937: 48

Idiodonus graeculus: DeLong, 1946a: 14

Cetexa graecula: Oman, 1949: 129

USNM (holotype); CAJAPE (other material)

Mexico (COAH) and USA 


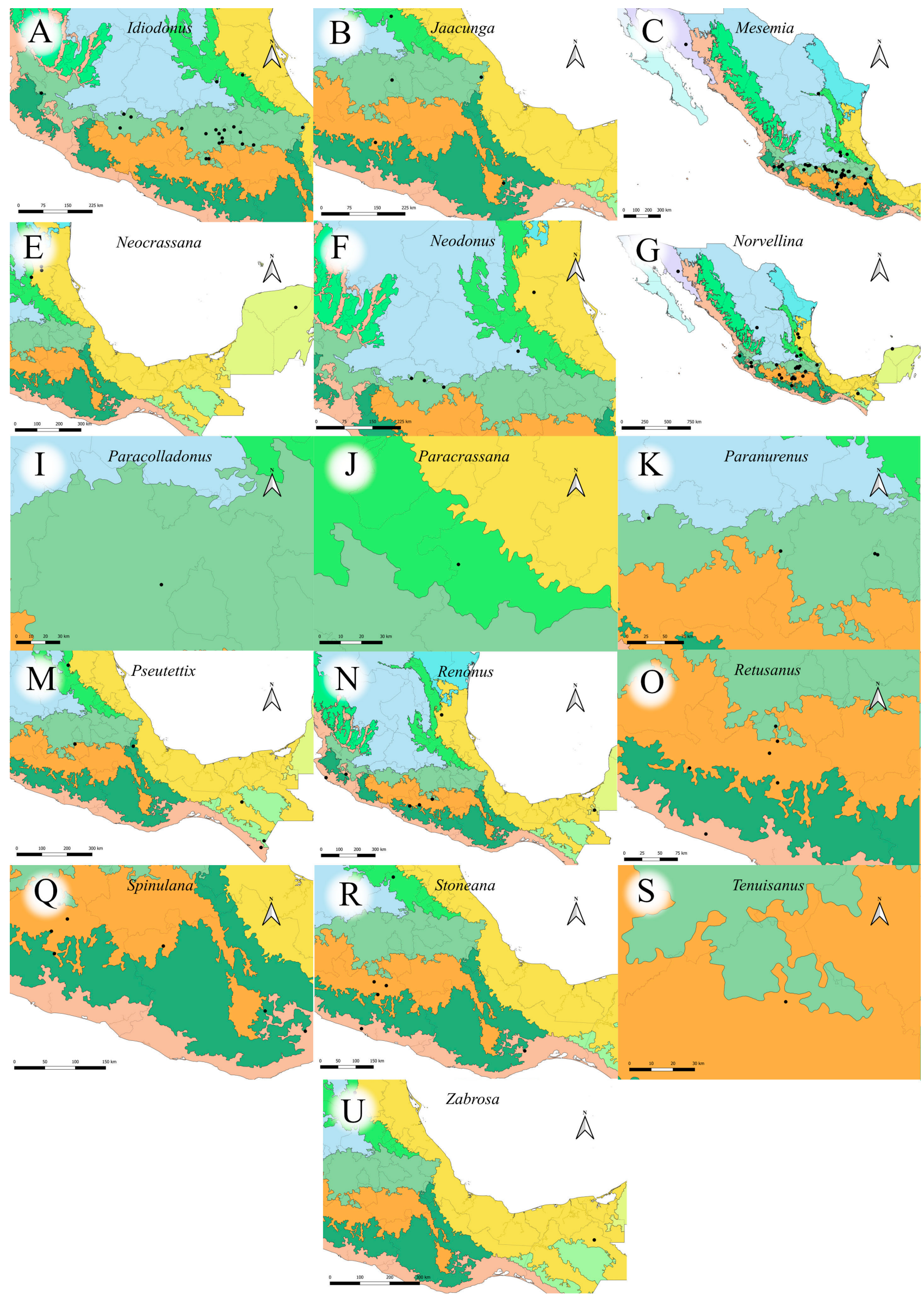

Fig. 5. Distributions of genera of Athysanini in Mexico (continued). 


\section{Genus Cocrassana Blocker \& Larsen, 1991}

Cocrassana Blocker \& Larsen, 1991: 124 (type species: Cocrassana riepmai Blocker \& Larsen, 1991)

Mexican Transition Zone and Neotropical; distribution in Mexico Fig. 4N

Pinedo-Escatel et al. (2018), DeLong (1959)

Host: Tripsacum pilosum; T. dactyloides; Zea perennis; Brachiaria plantaginea; Digitaria ciliaris; Cynodon plectostachyus; $C$. dactylon; Rhynchelytrum repens; Eleusine indica; Zea mays; Citrus $\times$ limon; Ficus carica.

Fig. $6 \mathrm{~S}$ and $\mathrm{Z}$

\section{Cocrassana sexvara (DeLong, 1959)}

Chlorotettix sexvarus DeLong, 1959: 326

Cocrassana sexvara: Pinedo-Escatel et al., 2018: 177

Cocrassana riepmai Blocker \& Larsen, 1991: 124 (synonymized by Pinedo-Escatel et al., 2018: 177)

OSUC (holotype and paratypes); CAJAPE (other material)

Endemic to Mexico (JAL; CAMP; SLP; VER; MIC; MOR)

\section{Genus Colladonus Ball, 1936}

Colladonus Ball, 1936: 57 (type species: Thamnotettix collaris Ball, 1902)

Conodonus Ball, 1936: 58

Friscananus Ball, 1936: 60

Hypospadianus Ribaut, 1942: 264

Sequoiatettix Bliven, 1955: 3

Coniferadonus Bliven, 1955: 4

Nearctic and Mexican Transition Zone; distribution in Mexico Fig. $4 \mathrm{O}$

Host: Pinus spp.

Oman (1949), Nielson (1988), DeLong (1946a, 1983)

Colladonus albocinctus (DeLong, 1946)

Idiodonus albocinctus DeLong, 1946a: 22

Idiodonus nigridens DeLong, 1946a: 29

Idiodonus sexpunctatus DeLong, 1983: 90

OSUC (holotype and paratypes)

Endemic to Mexico (EDOMEX; HGO; MIC; MOR; CDMX)

\section{Colladonus anademus (DeLong, 1946)}

Idiodonus anademus DeLong, 1946a: 29

Colladonus anademus: Nielson, 1957: 51

OSUC (holotype and paratypes)

Endemic to Mexico (EDOMEX)

\section{Colladonus beameri (Ball, 1937)}

Idiodonus beameri Ball, 1937: 28

Colladonus beameri: Oman, 1949: 125

Idiodonus marginatus DeLong, 1983: 90

USNM (holotype); CNIN and CEAM (other material)

Endemic to Mexico (MIC; EDOMEX; HGO; PUE; VER)

\section{Colladonus bicinctus (DeLong, 1946)}

Idiodonus bicinctus DeLong, 1946a: 18

Colladonus bicinctus: Nielson, 1988: 113

OSUC (holotype and paratype)

Endemic to Mexico (CDMX)

\section{Colladonus clathrus (DeLong, 1946)}

Idiodonus clathrus DeLong, 1946a: 18

Colladonus clathrus: Nielson, 1957: 51

Idiodonus turpiter DeLong, 1946a: 28 (synonymized by Nielson, 1988: 120)

OSUC (holotype and paratype)

Endemic to Mexico (PUE; EDOMEX)

Colladonus claustrus (DeLong, 1946)

Idiodonus claustrus DeLong, 1946a: 18
Colladonus claustrus: Nielson, 1957: 51

OSUC (holotype and paratype)

Endemic to Mexico (VER; CHIS)

Colladonus dampfi (DeLong, 1946)

Idiodonus dampfi DeLong, 1946a: 20

Colladonus dampfi: Nielson, 1957: 51

OSUC (holotype and paratypes)

Endemic to Mexico (VER; EDOMEX; MIC)

Colladonus fasciaticollis (Stål, 1864)

Jassus fasciaticollis Stål, 1864a: 86

Thamnotettix fasciaticollis: Van Duzee, 1892: 306

Colladonus fasciaticollis: DeLong \& Caldwell, 1937: 47

Idiodonus diserus DeLong, 1946a: 24 (synonymized by Nielson, 1957: 37)

Idiodonus pravus DeLong, 1946a: 24 (synonymized by Nielson, 1988: 109)

Idiodonus tubulus DeLong, 1946a: 22 (synonymized by Nielson, 1957: 37)

OSUC (other material)

Endemic to Mexico (EDOMEX; HGO; MIC; MOR; CDMX)

Colladonus incidus (DeLong, 1946)

Idiodonus incidus DeLong, 1946a: 29

Colladonus incidus: Nielson, 1988: 126

Idiodonus pallidus DeLong, 1983: 90

OSUC (holotype and paratype)

Endemic to Mexico (CDMX)

Colladonus titulus (DeLong, 1946)

Idiodonus titulus DeLong, 1946a: 24

Colladonus titulus: Nielson, 1957: 51

Idiodonus goodi DeLong, 1946a: 27

OSUC (holotype and paratypes)

Endemic to Mexico (CDMX; VER; EDOMEX)

Colladonus verecundus (DeLong, 1946)

Idiodonus verecundus DeLong, 1946a: 20

Colladonus verecundus: Nielson, 1957: 51

Idiodonus acus DeLong, 1946a: 20

Idiodonus mexicanus DeLong, 1946a: 27

OSUC (holotype and paratypes)

Endemic to Mexico (EDOMEX; MIC; MOR)

Genus Comayagua Linnavuori \& DeLong, 1978

Comayagua Linnavuori \& DeLong, 1978: 206 (type species: Comayagua taeniata Linnavuori \& DeLong, 1978)

Mexican Transition Zone and Neotropical; distribution in Mexico Fig. 4P

Fig. 6T and Aa

Comayagua taeniata Linnavuori \& DeLong, 1978

Comayagua taeniata Linnavuori \& DeLong, 1978: 208

OSUC (holotype and paratypes); CAJAPE (other material)

Mexico (JAL; OAX) and Honduras

Genus Conversana DeLong, 1967

Conversana DeLong, 1967d: 266 (type species: Conversana reversa DeLong, 1967)

Nearctic, Mexican Transition Zone, and Neotropical; distribution in Mexico Fig. 4Q

Fig. $6 \mathrm{U}$ and $\mathrm{Bb}$

Conversana angustata DeLong, 1967

Conversana angustata DeLong, 1967d: 268

OSUC (holotype and paratypes)

Endemic to Mexico (TAMPS) 

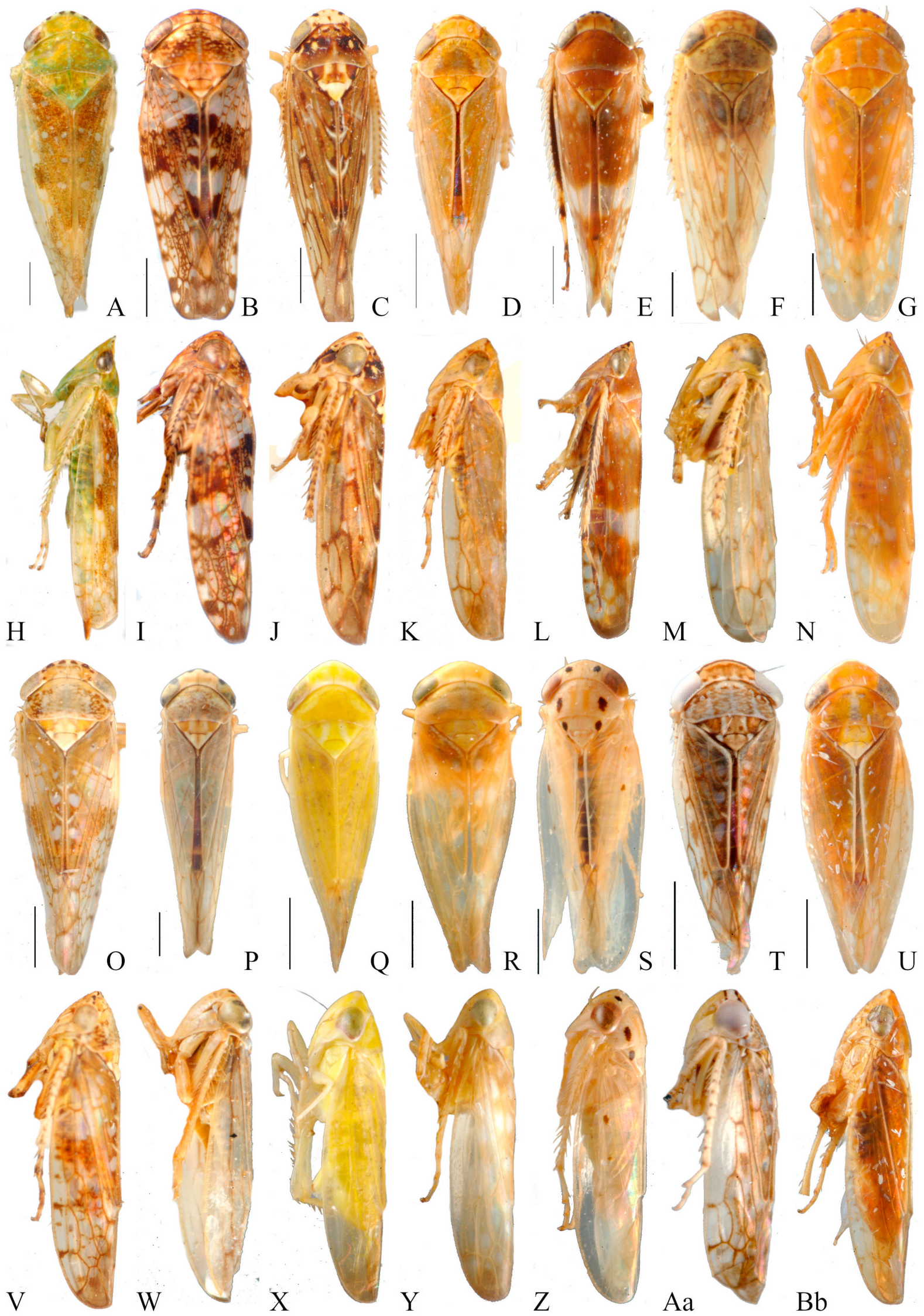

Fig. 6. Dorsal $(\mathrm{A}-\mathrm{G}, \mathrm{O}-\mathrm{U})$ and lateral $(\mathrm{H}-\mathrm{N}, \mathrm{V}-\mathrm{Bb})$ habitus of genera of Athysanini. $\mathrm{A}, \mathrm{H}-$ Acunasus nigriviridis; $\mathrm{B}, \mathrm{I}-$ Aligia alvona; $\mathrm{C}$, $\mathrm{J}$-Alladanus mexellus; D, K - Angulanus incisurus; F, L - Artucephalus fasciatus; F, M - Atanus coronatus*; G, N-Bandara johnsoni*; O, V - Bardana depressa; P, W - Bonneyana schwarzi; Q, X - Cahya chapadensis*; R, Y - Cetexa graecula; S, Z - Cocrassana sexvara; $\mathrm{T}, \mathrm{Aa}$ - Comayagua taeniata; $\mathrm{U}, \mathrm{Bb}$ - Conversana reversa. Asterisk indicates that the genus occurs in Mexico but not the species photographed. Scale bar $1 \mathrm{~mm}$. 


\section{Conversana conversa DeLong, 1967}

Conversana conversa DeLong, 1967d: 266

OSUC (holotype and paratypes)

Endemic to Mexico (PUE; MOR; CDMX)

Conversana reversa DeLong, 1967

Conversana reversa DeLong, 1967d: 267

OSUC (holotype and paratypes)

Endemic to Mexico (TAMPS)

Genus Costamia DeLong, 1946

Costamia DeLong, 1946b: 82 (type species: Costamia venosa DeLong, 1946)

Neotropical; distribution in Mexico Fig. 4R

Fig. 7A and $\mathrm{H}$

Costamia venosa DeLong, 1946

Costamia venosa DeLong, 1946b: 82

OSUC (holotype and paratypes); CAJAPE (other material)

Endemic to Mexico (GRO; OAX)

Genus Cozadanus DeLong \& Harlan, 1968

Cozadanus DeLong \& Harlan, 1968: 150 (type species: Cozadanus globosus DeLong \& Harlan, 1968)

Mexican Transition Zone; distribution in Mexico Fig. $4 \mathrm{~S}$

Fig. 7B and I

Cozadanus globosus DeLong \& Harlan, 1968

Cozadanus globosus DeLong \& Harlan, 1968: 150

OSUC (holotype)

Endemic to Mexico (GRO)

Cozadanus serratus DeLong \& Harlan, 1968

Cozadanus serratus DeLong \& Harlan, 1968: 150

OSUC (holotype)

Endemic to Mexico (MIC)

Genus Crassana DeLong \& Hershberger, 1947

Crassana DeLong \& Hershberger, 1947b: 76 (type species: Eutettix goniana Ball, 1931)

Nearctic, Mexican Transition Zone, and Neotropical; distribution in Mexico Fig. 4T

Blocker \& Larsen (1991)

Fig. $7 \mathrm{C}$ and $\mathrm{J}$

Subgenus Crassana (Crassana) DeLong \& Hershberger, 1947

Crassana (Crassana) DeLong \& Hershberger, 1947b: 76 (type species: Eutettix goniana Ball, 1931)

Crassana (Crassana) goniana (Ball, 1931)

Eutettix goniana Ball, 1931: 1

Crassana goniana: DeLong \& Hershberger, 1947b: 76

USNM (holotype); OSUC, CAJAPE, and CNIN (other material)

Mexico (GRO; PUE; MIC; TAMPS; SIN; MOR; SLP; CDMX) and USA

Subgenus Crassana (Macrasana) DeLong \& Hershberger, 1947

Crassana (Macrasana) DeLong \& Hershberger, 1947b: 78 (type species: Crassana marginella DeLong \& Hershberger, 1947)

Crassana (Macrasana) marginella DeLong \& Hershberger, 1947

Crassana (Macrasana) marginella DeLong \& Hershberger, 1947b: 78

OSUC (holotype); CAJAPE (other material)

Endemic to Mexico (GRO; OAX; NL)

Genus Dampfiana DeLong \& Hershberger, 1948

Dampfiana DeLong \& Hershberger, 1948a: 229 (type species:

Dampfiana deserta DeLong \& Hershberger, 1948)
Neotropical; distribution in Mexico Fig. 4U

Fig. 7D and $\mathrm{K}$

Dampfiana deserta DeLong \& Hershberger, 1948

Dampfiana deserta DeLong \& Hershberger, 1948a: 229

OSUC (holotype and paratypes)

Endemic to Mexico (GRO; TAMPS)

Genus Deltorynchus DeLong, 1943

Deltorynchus DeLong, 1943b: 79 (type species: Deltorynchus quadrinotus DeLong, 1943)

Neotropical; distribution in Mexico Fig. 4V

Fig. 7E and L

Deltorynchus quadrinotus DeLong, 1943

Deltorynchus quadrinotus DeLong, 1943b: 79

OSUC (holotype and paratypes)

Endemic to Mexico (GRO)

Deltorynchus spinosus DeLong, 1943

Deltorynchus spinosus DeLong, 1943b: 80

OSUC (holotype)

Endemic to Mexico (MOR)

Genus Duocrassana Pinedo-Escatel, Dietrich \& Zahniser, 2016

Duocrassana Pinedo-Escatel, Dietrich \& Zahniser, 2016: 580 (type species: Doucrassana longula Pinedo-Escatel, Dietrich \& Zahniser, 2016)

Neotropical; distribution in Mexico Fig. 4V

Duocrassana longula Pinedo-Escatel, Dietrich \& Zahniser, 2016

Duocrassana longula Pinedo-Escatel, Dietrich \& Zahniser, 2016: 586

INHS (holotype); CZUG (paratype); CAJAPE (other material)

Endemic to Mexico (OAX; YUC)

Genus Eusora Oman, 1949

Eusora Oman, 1949: 137 (type species: Eutettix animana Ball, 1909)

Nearctic; distribution in Mexico Fig. 4Y

Fig. 7F and $\mathrm{M}$

Eusora fenestrata (Ball, 1902)

Eutettix fenestrata Ball, 1902: 12

Eutettix (Mesamia) fenestrata: Ball, 1907: 65

Bandara fenestrata: Ball, 1931: 93

Eusora fenestrata: Oman, 1949: 137

USNM (holotype); CAJAPE (other material)

Mexico (SON) and USA

Genus Eutettix Van Duzee, 1892

Eutettix Van Duzee, 1892: 307 (type species: Thamnotettix lurida Van Duzee, 1890)

Nearctic, Mexican Transition Zone, and Neotropical; distribution in Mexico Fig. 4Z

Oman (1949), DeLong \& Harlan (1968), Hepner (1942b), DeLong (1980)

Fig. $8 \mathrm{G}$ and $\mathrm{N}$

Subgenus Eutettix (Eutettix) Van Duzee, 1892

Eutettix Van Duzee, 1892: 307 (type species: Thamnotettix luridus Van Duzee, 1890)

Eutettix (Eutettix): Ball, 1907: 31

Eutettix (Eutettix) alvadus DeLong \& Harlan, 1968

Eutettix (Eutettix) alvadus DeLong \& Harlan, 1968: 142

OSUC (holotype and paratypes)

Endemic to Mexico (HGO) 

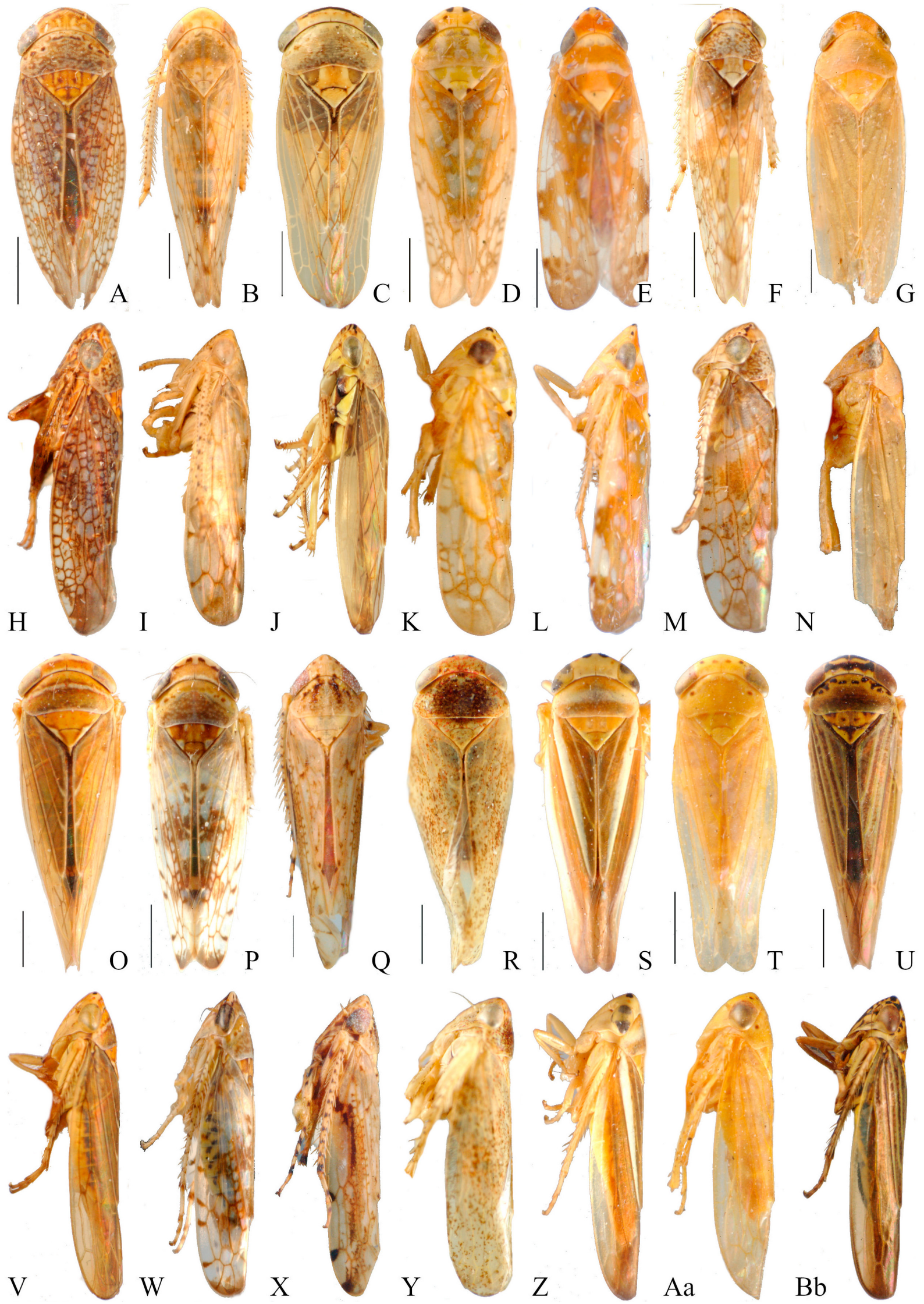

Fig. 7. Dorsal $(A-G, O-U)$ and lateral $(\mathrm{H}-\mathrm{N}, \mathrm{V}-\mathrm{Bb})$ habitus of genera of Athysanini. A, $\mathrm{H}$ - Costamia venosa; $\mathrm{B}, \mathrm{I}-\mathrm{Cozadanus} \mathrm{serratus;}$ C, J - Crassana (Crassana) goniana; D, K - Dampfiana deserta; E, L - Deltorynchus quadrinotus; F, M - Eusora fenestrata; G, N - Excavanus angustus; O, V - Jaacunga spatulata; P, W - Mesamia nigridorsum*; Q, X - Mexicananus levis; R, Y - Neodonus piperatus; S, $\mathrm{Z}$ - Idiodonus kennicotti; $\mathrm{T}, \mathrm{Aa}$ - Ollarianus balli*; $\mathrm{U}, \mathrm{Bb}$ - Paracolladonus insculptus. Asterisk indicates that the genus occurs in Mexico but not the species photographed. Scale bar $1 \mathrm{~mm}$. 
Eutettix (Eutettix) chelatus DeLong \& Harlan, 1968

Eutettix chelatus DeLong \& Harlan, 1968: 142

OSUC (holotype); OSUC and USNM (paratypes)

Endemic to Mexico (EDOMEX; CDMX; VER)

Eutettix (Eutettix) contorqus DeLong \& Harlan, 1968

Eutettix (Eutettix) contorqus DeLong \& Harlan, 1968: 140 OSUC (holotype); OSUC and USNM (paratypes)

Endemic to Mexico (MIC; JAL; HGO)

Eutettix (Eutettix) divergens DeLong \& Harlan, 1968 Eutettix (Eutettix) divergens DeLong \& Harlan, 1968: 146 OSUC (holotype and paratypes)

Endemic to Mexico (CDMX)

Eutettix (Eutettix) guevarai DeLong \& Harlan, 1968

Eutettix (Eutettix) guevarai DeLong \& Harlan, 1968: 146 OSUC (holotype)

Endemic to Mexico (PUE)

Eutettix (Eutettix) harlani Zahniser, McKamey \& Dmitriev, 2012

Eutettix (Eutettix) dentatus DeLong \& Harlan, 1968: 141 (preoccupied)

Eutettix (Eutettix) harlani Zahniser, McKamey \& Dmitriev, 2012: 357

OSUC (holotype and paratypes); OSUC and CNIN (other material)

Endemic to Mexico (MIC; CDMX; MIC)

Eutettix (Eutettix) krameri DeLong \& Harlan, 1968

Eutettix (Eutettix) krameri DeLong \& Harlan, 1968: 146

USNM (holotype)

Endemic to Mexico (HGO)

Eutettix (Eutettix) lanceolatus DeLong \& Harlan, 1968

Eutettix (Eutettix) lanceolatus DeLong \& Harlan, 1968: 141

OSUC (holotype and paratypes)

Endemic to Mexico (PUE)

Eutettix (Eutettix) ortegai DeLong \& Harlan, 1968

Eutettix (Eutettix) ortegai DeLong \& Harlan, 1968: 147

OSUC (holotype)

Endemic to Mexico (PUE)

Eutettix (Eutettix) pedus DeLong \& Harlan, 1968

Eutettix (Eutettix) pedus DeLong \& Harlan, 1968: 144

OSUC (holotype); OSUC and USNM (other material)

Endemic to Mexico (EDOMEX; MIC; PUE; MOR; CDMX)

Eutettix (Eutettix) pictus Van Duzee, 1892

Eutettix pictus Van Duzee, 1892: 301

Eutettix picta: Van Duzee, 1894: 297

Eutettix magnus Osborn, 1900: 395

Eutettix (Eutettix) subaenea picta: Ball, 1907: 31

Eutettix (Eutettix) picta: Ball, 1907: 73

OSUC (paratype)

Mexico (NL) and USA

Eutettix (Eutettix) planus Hepner, 1942

Eutettix (Eutettix) planus Hepner, 1942: 260

KUNHM (holotype); USNM and OSUC (paratypes)

Mexico (SON) and USA

Eutettix (Eutettix) spinus DeLong \& Harlan, 1968

Eutettix (Eutettix) spinus DeLong \& Harlan, 1968: 144

OSUC (holotype and paratypes)

Endemic to Mexico (HGO; CDMX; EDOMEX)

Eutettix (Eutettix) transversus DeLong \& Harlan, 1968

Eutettix (Eutettix) transversus DeLong \& Harlan, 1968: 147
OSUC (holotype and paratypes)

Endemic to Mexico (MIC; GRO)

Subgenus Eutettix (Guadlera) DeLong, 1980

Eutettix (Guadlera) DeLong, 1980: 65 (type species: Eutettix discapa DeLong, 1980)

Eutettix (Guadlera) copula DeLong, 1980

Eutettix (Guadlera) copula DeLong, 1980: 65

OSUC (holotype)

Endemic to Mexico (CDMX)

Eutettix (Guadlera) discapa DeLong, 1980

Eutettix (Guadlera) discapa DeLong, 1980: 65

OSUC (holotype and paratypes)

Endemic to Mexico (MIC; EDOMEX; CDMX)

Eutettix (Guadlera) placida DeLong, 1980

Eutettix (Guadlera) placida DeLong, 1980: 65

OSUC (holotype and paratypes)

Endemic to Mexico (MIC)

Genus Excavanus DeLong, 1946

Excavanus DeLong, 1946c: 446 (type species: Excavanus angustus DeLong, 1946)

Neotropical; distribution in Mexico Fig. 4K

Fig. 7G and $\mathrm{N}$

Excavanus angustus DeLong, 1946

Excavanus angustus DeLong, 1946c: 446

OSUC (holotype)

Endemic to Mexico (GRO)

Genus Idiodonus Ball, 1936

Idiodonus Ball, 1936: 57 (type species: Jassus kennicotti Uhler, 1864)

Idiodonus (Idiodonus): DeLong, 1946a: 30

Phlepsius (Josanus) DeLong, 1938: 244

Orolix Ribaut, 1942: 267

Nearctic and Mexican Transition Zone; distribution in Mexico Fig. 5A

Oman (1949), DeLong (1983, 1984)

Host: Pinus spp.; Quercus spp.

Fig. $7 \mathrm{~S}$ and $\mathrm{Z}$

Idiodonus beamerellus DeLong, 1983

Idiodonus beameri DeLong, 1946a: 92 (preoccupied)

Idiodonus beamerellus DeLong, 1983: 92

OSUC (holotype, paratypes, and other material)

Endemic to Mexico (MIC; HGO)

Idiodonus copulus DeLong, 1946

Idiodonus copulus DeLong, 1946a: 16

OSUC (holotype and paratypes)

Endemic to Mexico (MIC; GRO; VER)

Idiodonus edentulus DeLong, 1946

Idiodonus edentulus DeLong, 1946a: 25

OSUC (holotype and paratypes)

Endemic to Mexico (EDOMEX)

Idiodonus excavatus DeLong, 1946

Idiodonus excavatus DeLong, 1946a: 25

OSUC (holotype and paratypes)

Endemic to Mexico (HGO; EDOMEX)

Idiodonus plummeri DeLong, 1946

Idiodonus plummeri DeLong, 1946a: 25

Idiodonus bakeri DeLong, 1946a: 26

OSUC (holotype and paratypes)

Endemic to Mexico (EDOMEX; MOR) 

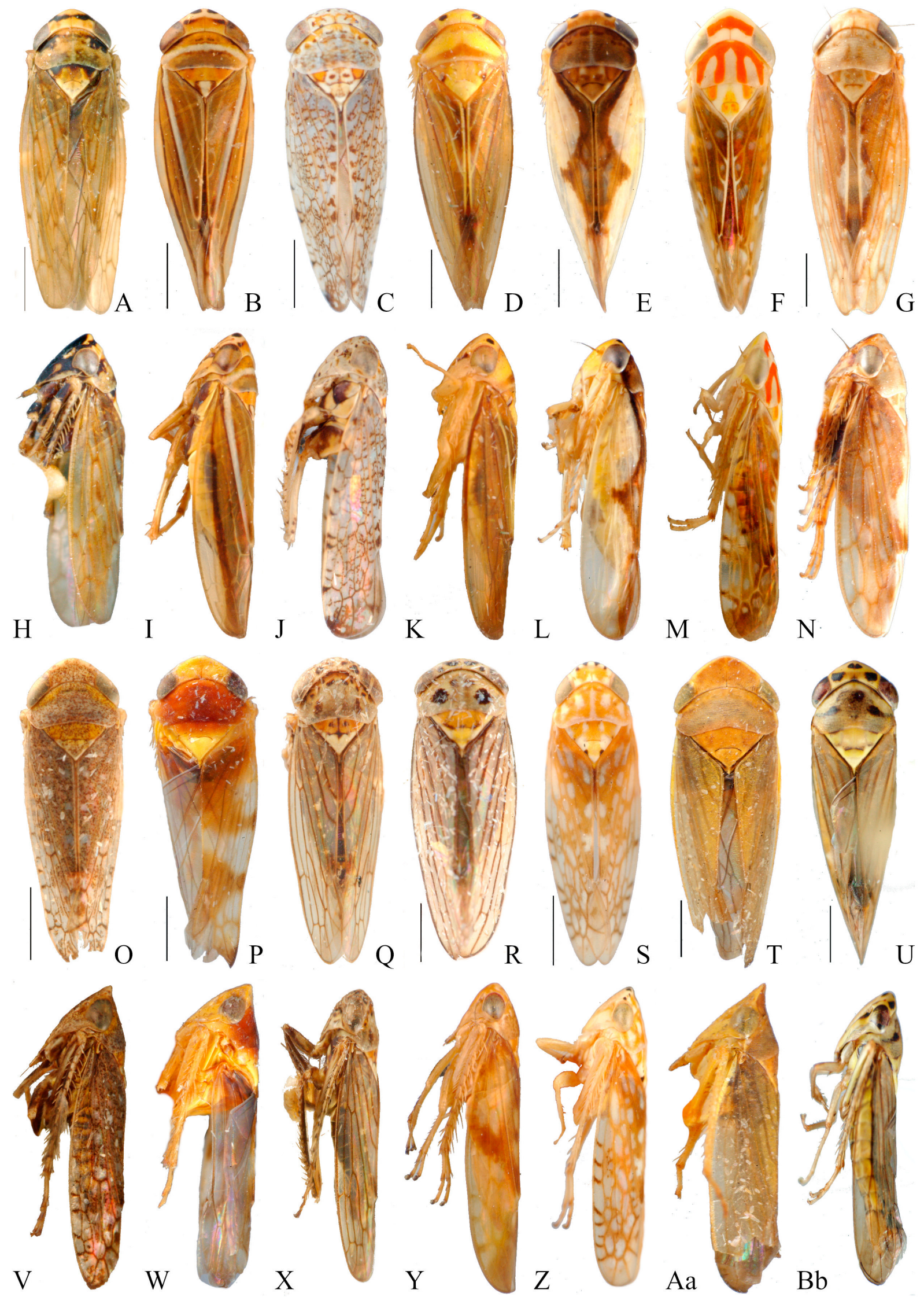

Fig. 8. Dorsal $(\mathrm{A}-\mathrm{G}, \mathrm{O}-\mathrm{U})$ and lateral $(\mathrm{H}-\mathrm{N}, \mathrm{V}-\mathrm{Bb})$ habitus of genera of Athysanini. $\mathrm{A}, \mathrm{H}-$ Paracrassana nigrifrons; $\mathrm{B}, \mathrm{I}-\mathrm{Paranurenus}$ latidens; C, J - Pseudaligia nigropunctata; D, K - Pseutettix binotata; E, L - Neocrassana undata*; F, M - Renonus rubraviridis; G, N - Eutettix luridus*; O, V - Retusanus luteus; P, W - Sanuca badia; Q, X - Spinulana varigata; R - Usanus stonei; Y - Carelmapu aureonitens*; $\mathrm{S}, \mathrm{Z}$ - Stoneana marthae; T, Aa - Tenuisanus costatus; U, Bb - Zabrosa amazonensis. Asterisk indicates that the genus occurs in Mexico but not the species photographed. Scale bar $1 \mathrm{~mm}$. 


\section{Idiodonus wickhami Ball, 1937}

Idiodonus wickhami Ball, 1937: 27

USNM (holotype and paratypes); OSUC, CAJAPE, INHS,

CNIN, CEAM, and CZUG (other material)

Mexico (PUE; EDOMEX; CDMX; PUE; MIC) and USA

Genus Jaacunga Nielson, 1988

Jaacunga Nielson, 1988: 129 (type species: Idiodonus vinculus DeLong, 1946)

Nearctic and Mexican Transition Zone; distribution in Mexico Fig. 5B

DeLong (1946a)

Fig. $7 \mathrm{O}$ and $\mathrm{V}$

Jaacunga spatulata (DeLong, 1946)

Idiodonus spatulatus DeLong, 1946a: 15

Idiodonus rubellus DeLong, 1946a: 15

OSUC (holotype and paratypes)

Endemic to Mexico (CDMX)

\section{Jaacunga vincula (DeLong, 1946)}

Idiodonus vinculus DeLong, 1946a: 15

OSUC (holotype and paratypes)

Endemic to Mexico (HGO; GRO; CDMX; VER)

Genus Mesamia Ball, 1907

Eutettix (Mesamia) Ball, 1907: 59 (type species: Eutettix nigridorsum Ball, 1907)

Mesamia: Smith, 1910: 103

Nearctic, Mexican Transition Zone, and Neotropical; distribution in Mexico Fig. 5C

Oman (1949), DeLong \& Hershberger (1947c), DeLong (1980)

Fig. 7P and W

Mesamia alta DeLong \& Hershberger, 1947

Mesamia alta DeLong \& Hershberger, 1947: 260

OSUC (holotype and paratypes)

Endemic to Mexico (MIC; PUE; GRO)

Mesamia bifurcata DeLong \& Hershberger, 1947

Mesamia bifurcata DeLong \& Hershberger, 1947: 258

OSUC (holotype); OSUC and USNM (paratypes)

Endemic to Mexico (PUE; MIC; CDMX; EDOMEX; MOR; VER; HGO)

Mesamia divisa DeLong \& Hershberger, 1947

Mesamia divisa DeLong \& Hershberger, 1947: 262

OSUC (holotype and paratypes)

Endemic to Mexico (GRO; HGO; VER; MIC; JAL; COAH)

Mesamia forcipata DeLong, 1980

Mesamia forcipata DeLong, 1980: 66

OSUC (holotype)

Endemic to Mexico (GRO)

Mesamia frigida DeLong \& Hershberger, 1947

Mesamia frigida DeLong \& Hershberger, 1947: 265

OSUC (holotype)

Mexico (EDOMEX) and USA

Mesamia interrupta DeLong \& Hershberger, 1947

Mesamia interrupta DeLong \& Hershberger, 1947: 263

OSUC (holotype and paratypes)

Endemic to Mexico (JAL; CDMX)

Mesamia montana DeLong \& Hershberger, 1947

Mesamia montana DeLong \& Hershberger, 1947: 262

OSUC (holotype and paratypes)

Mexico (MIC) and USA
Mesamia orizaba Ball, 1931

Mesamia orizaba Ball, 1931: 92

USNM (holotype and paratypes)

Endemic to Mexico (VER; MIC; GRO; PUE; CDMX; HGO)

Mesamia puebla DeLong \& Hershberger, 1947

Mesamia puebla DeLong \& Hershberger, 1947: 264

OSUC (holotype)

Endemic to Mexico (PUE)

Mesamia ruptura DeLong, 1980

Mesamia ruptura DeLong, 1980: 66

OSUC (holotype)

Endemic to Mexico (GRO)

Mesamia separata DeLong \& Hershberger, 1947

Mesamia separata DeLong \& Hershberger, 1947: 263

OSUC (holotype and paratypes)

Endemic to Mexico (VER; GRO; PUE; HGO; JAL)

Mesamia tarbela Ball, 1931

Mesamia tarbela Ball, 1931: 91

USNM (holotype)

Mexico (SON) and USA

Genus Mexicananus DeLong, 1944

Mexicananus DeLong, 1944a: 89 (type species: Mexicananus levis DeLong, 1944)

Neotropical; distribution in Mexico Fig. 5D

Fig. 7Q and $\mathrm{X}$

\section{Mexicananus levis DeLong, 1944}

Mexicananus levis DeLong, 1944a: 89

OSUC (holotype); USNM (other material)

Endemic to Mexico (CHIS; VER)

Genus Neocrassana Linnavuori, 1959

Neocrassana Linnavuori, 1959: 286 (type species: Neocrassana undata Linnavuori, 1959)

Neotropical; distribution in Mexico Fig. 5E

Blocker \& Larsen (1991)

Fig. 8E and L

Neocrassana punctiger Linnavuori, 1959

Neocrassana punctiger Linnavuori, 1959: 287

USNM (holotype and paratypes)

Mexico (SLP; YUC) and Panama

Genus Neodonus DeLong \& Hershberger, 1948

Neodonus DeLong \& Hershberger, 1948b: 159 (type species: Neodonus piperatus DeLong \& Hershberger, 1948)

Nearctic, Mexican Transition Zone, and Neotropical; distribution in Mexico Fig. 5F

Fig. 7R and $\mathrm{Y}$

Neodonus piperatus DeLong \& Hershberger, 1948

Neodonus piperatus DeLong \& Hershberger, 1948b: 159

OSUC (holotype and paratypes)

Endemic to Mexico (MIC; HGO; SLP)

Genus Norvellina Ball, 1931

Norvellina Ball, 1931: 2 (type species: Eutettix mildredae Ball, 1901)

Nearctic, Mexican Transition Zone, and Neotropical; distribution in Mexico Fig. 5G

Oman (1949), Kramer \& DeLong (1969), Lindsay (1939)

Norvellina acuspina Kramer \& DeLong, 1969

Norvellina acuspina Kramer \& DeLong, 1969: 115

OSUC (holotype and paratypes)

Endemic to Mexico (CHIS) 


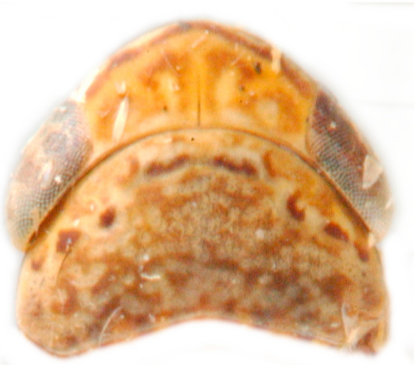

A

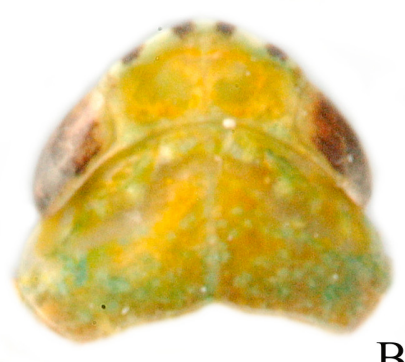

$\mathrm{B}$
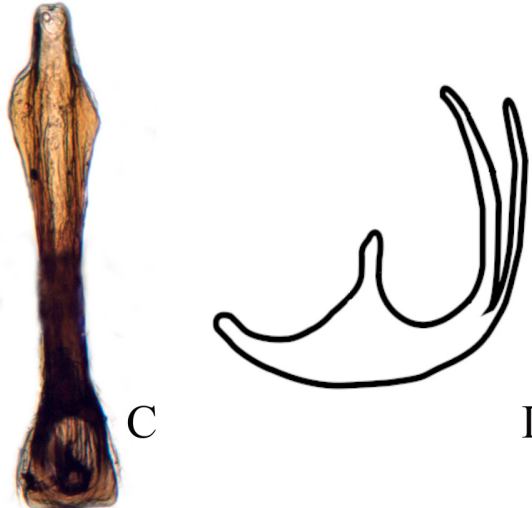

$\mathrm{D}$
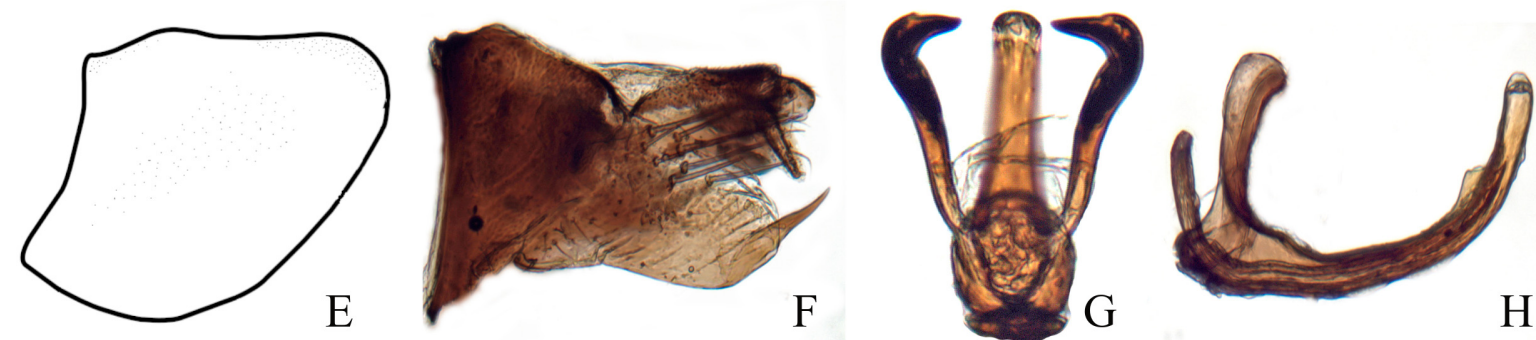

$\mathrm{H}$
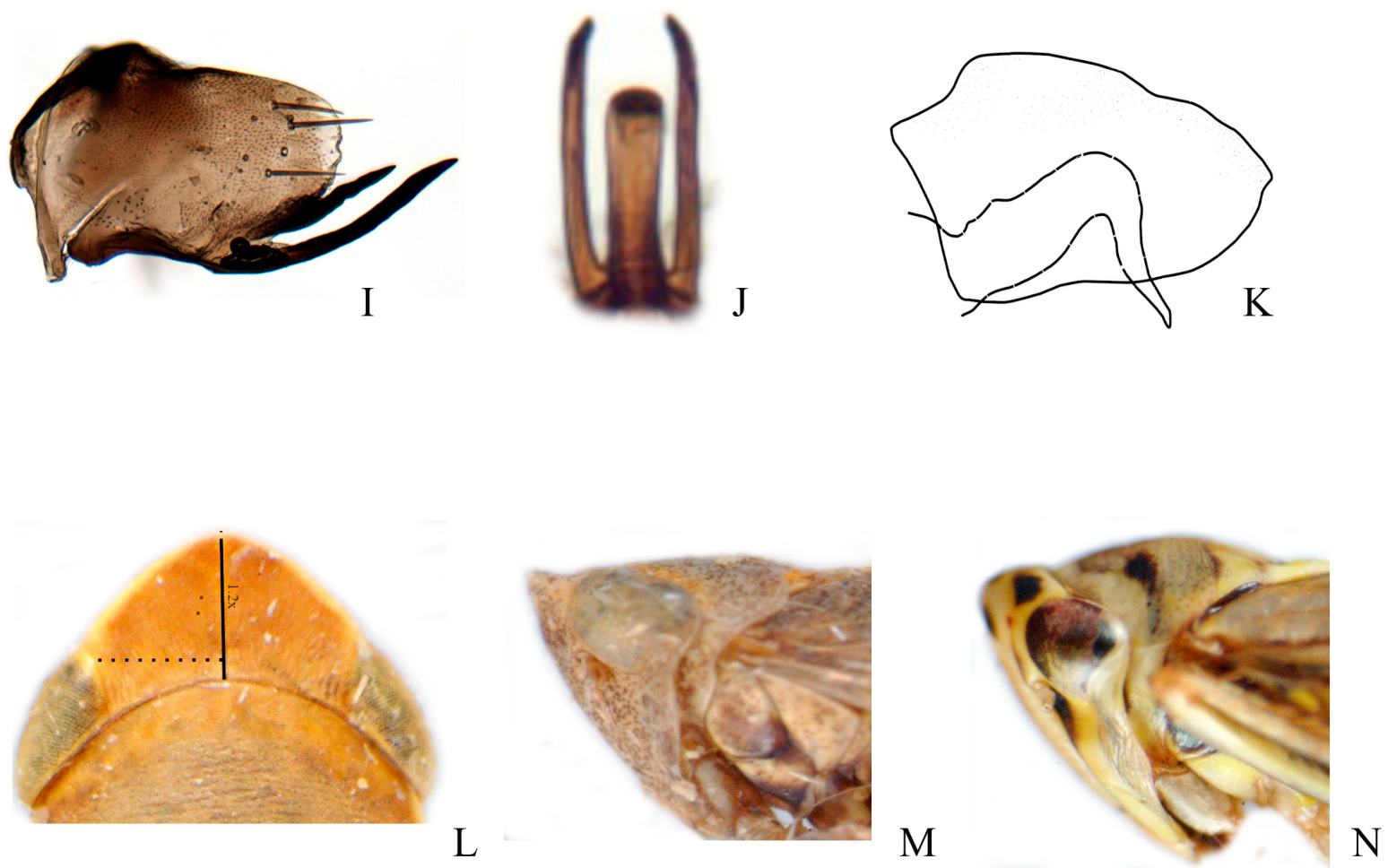

Fig. 9. Morphological features of Mexican Athysanini. A - head of Costamia venosa, dorsal view; B - head of Acunasus nigriviridis, dorsal view; C - aedeagus of Paracrassana nigrifrons, ventral view; D - aedeagus of Mesamia divisa, lateral view; $\mathrm{E}-$ pygofer of $C$. venosa, lateral view; F - pygofer of Bardana depressa, lateral view; G - aedeagus of Angulanus incisurus, anterior view; $\mathrm{H}$ - aedeagus of $P$. nigrifrons, lateral view; I - pygofer of Sanuca badia, lateral view; J - aedeagus of $B$. depressa, posterior view; $\mathrm{K}$ - pygofer of Cozadanus serratus, lateral view; $\mathrm{L}$ - head of Tenuisanus costatus, dorsal view; $\mathrm{M}$ - head of Retusanus luteus, lateral view; $\mathrm{N}-\mathrm{head}$ of Zabrosa amazonensis, lateral view. 
Norvellina adunca Kramer \& DeLong, 1969

Norvellina adunca Kramer \& DeLong, 1969: 116

OSUC (holotype and paratypes)

Endemic to Mexico (TAMPS; GRO; CHIS)

\section{Norvellina cincta Kramer \& DeLong, 1969}

Norvellina cincta Kramer \& DeLong, 1969: 120

OSUC (holotype); CAJAPE (other material)

Endemic to Mexico (PUE; GRO)

Norvellina denotata Kramer \& DeLong, 1969

Norvellina denotata Kramer \& DeLong, 1969: 118

OSUC (holotype and paratypes)

Endemic to Mexico (CDMX; GRO; MIC; EDOMEX)

Norvellina forficata Kramer \& DeLong, 1969

Norvellina forficata Kramer \& DeLong, 1969: 118

OSUC (holotype and paratypes)

Endemic to Mexico (VER; PUE)

\section{Norvellina pulchella (Baker, 1896)}

Eutettix pulchella Baker, 1896: 24

Eutettix (Eutettix) pulchella: Ball, 1907: 33

Eutettix pulchellus: Van Duzee, 1916: 72

Norvellina pulchella: Ball, 1931: 3

Norvellina pulchellus: DeLong \& Caldwell, 1937: 40

CAJAPE, INHS, and OSUC (other material)

Mexico (BCS; HGO) and USA

Norvellina recepta Kramer \& DeLong, 1969

Norvellina recepta Kramer \& DeLong, 1969: 120

OSUC (holotype); INHS (other material)

Endemic to Mexico (SON; ZAC)

Norvellina spatulata DeLong, 1980

Norvellina spatulata DeLong, 1980: 69

OSUC (holotype)

Endemic to Mexico (HGO)

Norvellina uncata Kramer \& DeLong, 1969

Norvellina uncata Kramer \& DeLong, 1969: 116

OSUC (holotype and paratypes)

Endemic to Mexico (SLP; GRO; PUE; MIC; JAL)

Genus Ollarianus Ball, 1936

Ollarianus Ball, 1936: 59 (type species: Eutettix balli Van Duzee, 1907)

Nearctic, Mexican Transition Zone, and Neotropical; distribution in Mexico Fig. $5 \mathrm{H}$

DeLong (1944b), Oman (1949), Linnavuori (1959)

Fig. 7T and $\mathrm{Aa}$

Ollarianus advenus DeLong, 1980

Ollarianus advenus DeLong, 1980: 69

OSUC (holotype)

Endemic to Mexico (GRO)

Ollarianus armus (Ball, 1933)

Exitianus armus Ball, 1933: 227

Thamnotettix armus: Oman, 1938: 382

Ollarianus armus: DeLong, 1944b: 392

USNM (holotype); OSUC (other material)

Mexico (SON) and USA

Ollarianus bidentatus DeLong, 1944

Ollarianus bidentatus DeLong, 1944b: 397

OSUC (holotype and paratypes)

Endemic to Mexico (GRO; MOR; SLP)

Ollarianus insignis DeLong, 1944

Ollarianus insignis DeLong, 1944b: 396
OSUC (holotype and paratypes); CAJAPE and INHS (other material)

Mexico (VER; GRO; JAL; MOR; MIC; OAX) and Guatemala

Ollarianus kinoanus (Ball, 1936)

Exitianus kinoanus Ball, 1936: 72

Ollarianus kinoanus: DeLong \& Hershberger, 1947: 116

USNM (holotype); OSUC (other material)

Mexico (SON) and USA

Ollarianus lobatus DeLong, 1944

Ollarianus lobatus DeLong, 1944b: 397

OSUC (holotype)

Endemic to Mexico (GRO)

Ollarianus mexicanus DeLong, 1980

Ollarianus mexicanus DeLong, 1980: 70

OSUC (holotype and paratypes)

Endemic to Mexico (GRO; OAX; SON; TAMPS)

Ollarianus muesebecki DeLong, 1944

Ollarianus muesebecki DeLong, 1944b: 396

USNM (holotype and paratypes); CAJAPE (other material)

Endemic to Mexico (TAMPS; MIC; GRO)

Ollarianus sexmaculatus Linnavuori, 1959

Ollarianus sexmaculatus Linnavuori, 1959: 294

AMNH (holotype); HNHM (paratype)

Endemic to Mexico (GRO; YUC)

Ollarianus strictus (Ball, 1900)

Eutettix strictus Ball, 1900: 204

Eutettix (Eutettix) stricta: Ball, 1907: 32

Eutettix strictus: Van Duzee, 1916: 72

Chlorotettix minor DeLong, 1918: 6

Opsius strictus: Ball, 1931: 2

Ollarianus strictus: Ball, 1936: 322

Norvellina strictus: DeLong \& Caldwell, 1937: 40

Norvellina stricta: Lindsay, 1939: 171

USNM (holotype); OSUC, CAJAPE, INHS, CEAM, and CNIN (other material)

Mexico (GRO; OAX; SON; NL; JAL) and USA

Ollarianus tripartitus DeLong, 1944

Ollarianus tripartitus DeLong, 1944b: 396

Idiodonus albifrons DeLong, 1983: 89

OSUC (holotype and paratypes); INHS and CAJAPE (other material)

Endemic to Mexico (GRO)

Ollarianus vestigii DeLong, 1944

Ollarianus vestigii DeLong, 1944b: 398

OSUC (holotype and paratypes)

Endemic to Mexico (SLP)

Genus Paracolladonus Nielson, 1988

Paracolladonus Nielson, 1988: 127 (type species: Idiodonus insculptus DeLong, 1946)

Mexican Transition Zone; distribution in Mexico Fig. 5I

DeLong (1946a)

Fig. $7 \mathrm{U}$ and $\mathrm{Bb}$

Paracolladonus insculptus (DeLong, 1946)

Idiodonus insculptus DeLong, 1946a: 25

Paracolladonus insculptus: Nielson, 1988: 127

OSUC (holotype and paratypes)

Endemic to Mexico (EDOMEX)

Genus Paracrassana Nielson, 1988

Paracrassana Nielson, 1988: 132 (type species: Idiodonus nigrifrons DeLong, 1983) 
Mexican Transition Zone; distribution in Mexico Fig. 5J

DeLong (1983)

Fig. 8A and $\mathrm{H}$

Paracrassana nigrifrons (DeLong, 1983)

Idiodonus nigrifrons DeLong, 1983: 91

Paracrassana nigrifrons: Nielson, 1988: 132

OSUC (holotype)

Endemic to Mexico (PUE)

\section{Genus Paranurenus Nielson, 1988}

Paranurenus Nielson, 1988: 127 (type species: Idiodonus latidens DeLong, 1946)

Mexican Transition Zone; distribution in Mexico Fig. $5 \mathrm{~K}$

DeLong (1946a)

Fig. 8B and I

Paranurenus latidens (DeLong, 1946)

Idiodonus latidens DeLong, 1946a: 26

Paranurenus latidens: Nielson, 1988: 128

OSUC (holotype and paratypes)

Endemic to Mexico (MIC; CDMX)

Genus Pseudaligia Kramer \& DeLong, 1968

Pseudaligia Kramer \& DeLong, 1968: 171 (type species: Pseudaligia nigropunctata Kramer \& DeLong, 1968)

Mexican Transition Zone and Neotropical; distribution in Mexico Fig. 5L

Fig. $8 \mathrm{C}$ and $\mathrm{J}$

Pseudaligia albocincta Kramer \& DeLong, 1968

Pseudaligia albocincta Kramer \& DeLong, 1968: 172

OSUC (holotype)

Endemic to Mexico (GRO)

Pseudaligia nigropunctata Kramer \& DeLong, 1968

Pseudaligia nigropunctata Kramer \& DeLong, 1968: 171

OSUC (holotype); OSUC and USNM (paratypes)

Endemic to Mexico (HGO; GRO)

Genus Pseutettix DeLong, 1967

Pseutettix DeLong, 1967a: 210 (type species: Pseutettix mexicana [sic] DeLong, 1967)

Mexican Transition Zone and Neotropical; distribution in Mexico Fig. 5M

Fig. $8 \mathrm{D}$ and $\mathrm{K}$

Pseutettix binotatus DeLong, 1967

Pseutettix binotata DeLong, 1967a: 212

OSUC (holotype and paratypes)

Endemic to Mexico (SLP; CHIS)

Pseutettix mexicanus DeLong, 1967

Pseutettix mexicana DeLong, 1967a: 210

OSUC (holotype and paratypes)

Endemic to Mexico (VER; MOR)

Genus Renonus DeLong, 1959

Renonus DeLong, 1959: 325 (type species: Renonus rubraviridis DeLong, 1959)

Neotropical; distribution in Mexico Fig. $5 \mathrm{~N}$

Fig. 8F and $\mathrm{M}$

Renonus rubraviridis DeLong, 1959

Renonus rubraviridis DeLong, 1959: 326

OSUC (holotype and paratypes)

Endemic to Mexico (JAL; GRO; TAB; TAMPS)

Genus Retusanus DeLong, 1945

Retusanus DeLong, 1945c: 135 (type species: Retusanus punctatus DeLong, 1945)

Neotropical; distribution in Mexico Fig. $5 \mathrm{O}$
Fig. $8 \mathrm{O}$ and $\mathrm{V}$

Retusanus apicatus DeLong, 1945

Retusanus apicatus DeLong, 1945c: 138

OSUC (holotype)

Endemic to Mexico (GRO)

Retusanus irroratus DeLong, 1945

Retusanus irroratus DeLong, 1945c: 140

OSUC (holotype)

Endemic to Mexico (GRO)

Retusanus luteus DeLong, 1945

Retusanus luteus DeLong, 1945c: 136

OSUC (holotype)

Endemic to Mexico (GRO)

Retusanus pulverus DeLong, 1945

Retusanus pulverus DeLong, 1945c: 136

OSUC (holotype)

Endemic to Mexico (GRO)

Retusanus punctatus DeLong, 1945

Retusanus punctatus DeLong, 1945c: 135

OSUC (holotype and paratypes); CAJAPE and INHS (other material)

Endemic to Mexico (GRO)

Genus Sanuca DeLong, 1980

Sanuca DeLong, 1980: 66 (type species: Sanuca badia DeLong, 1980)

Neotropical; distribution in Mexico Fig. 5P

Fig. 8P and W

Sanuca badia DeLong, 1980

Sanuca badia DeLong, 1980: 66

OSUC (holotype)

Endemic to Mexico (GRO)

Genus Spinulana DeLong, 1967

Spinulana DeLong, 1967b: 20 (type species: Spinulana varigata DeLong, 1967)

Neotropical and Mexican Transition Zone; distribution in Mexico Fig. 5Q

Fig. 8Q and $\mathrm{X}$

Spinulana spinosa DeLong, 1967

Spinulana spinosa DeLong, 1967b: 20

OSUC (holotype)

Endemic to Mexico (GRO)

Spinulana varigata DeLong, 1967

Spinulana varigata DeLong, 1967b: 21

OSUC (holotype and paratypes)

Endemic to Mexico (GRO)

Spinulana josefinae Pinedo-Escatel, sp. $\mathbf{n}$.

INHS (holotype); CAJAPE (paratype)

Endemic to Mexico (OAX)

Genus Stoneana DeLong, 1943

Stoneana DeLong, 1943c: 448 (type species: Stoneana marthae DeLong, 1943)

Neotropical and Mexican Transition Zone; distribution in Mexico Fig. 5R

Fig. $8 \mathrm{~S}$ and $\mathrm{Z}$

Stoneana balli DeLong, 1943

Stoneana balli DeLong, 1943c: 449

USNM (holotype); USNM and OSUC (paratypes); CAJAPE (other material)

Endemic to Mexico (GRO) 


\section{Stoneana marthae DeLong, 1943}

Stoneana marthae DeLong, 1943c: 448

USNM (holotype); USNM and OSUC (paratypes); CAJAPE (other material)

Endemic to Mexico (GRO)

\section{Stoneana separata DeLong, 1943}

Stoneana separatus DeLong, 1943c: 450

OSUC (holotype and paratypes)

Endemic to Mexico (GRO)

\section{Genus Tenuisanus DeLong, 1944}

Tenuisanus DeLong, 1944c: 73 (type species: Tenuisanus costatus DeLong, 1944)

Neotropical; distribution in Mexico Fig. 5S

Fig. 8T and Aa

Tenuisanus costatus DeLong, 1944

Tenuisanus costatus DeLong, 1944c: 73

OSUC (holotype)

Endemic to Mexico (GRO)

\section{Genus Usanus DeLong, 1947}

Usanus DeLong, 1947: 110 (type species: Usanus stonei DeLong, 1947)

Devolana DeLong, 1967c: 22

Neotropical; distribution in Mexico Fig. 5T

Aguilar-Pérez et al. (2019), Pinedo-Escatel \& Dietrich (2020a)

Fig. 8R

\section{Usanus igualaensis Pinedo-Escatel \& Dietrich, 2020}

Usanu igualaensis Pinedo-Escatel \& Dietrich, 2020a: 570

OSUC (holotype)

Endemic to Mexico (GRO)

Usanus stonei DeLong, 1947

Usanus stonei DeLong, 1947: 110

Devolana hemicycla DeLong, 1967c: 23

OSUC (holotype)

Endemic to Mexico (GRO)

\section{Usanus tuxcacuensis (Pinedo-Escatel \& Aguilar-Pérez,}

2019)

Devolana tuxcacuensis Pinedo-Escatel \& Aguilar-Pérez, 2019: 2042

Usanus tuxcacuensis: Pinedo-Escatel \& Dietrich, 2020a: 572

OSUC (holotype); CAJAPE (paratype)

Endemic to Mexico (JAL)

Usanus xajxayakamej (Pinedo-Escatel, 2019)

Devolana xajxayakamej Pinedo-Escatel, 2019: 2050

Usanus xajxayakamej: Pinedo-Escatel \& Dietrich, 2020a: 575

OSUC (holotype)

Endemic to Mexico (GRO)

Usanus xochipalensis Pinedo-Escatel \& Dietrich, 2020

Usanus xochipalensis Pinedo-Escatel \& Dietrich, 2020a: 572

TAMU (holotype)

Endemic to Mexico (GRO)

Usanus youajla (Pinedo-Escatel, 2019)

Devolana youajla Pinedo-Escatel, 2019: 2046

Usanus youajla: Pinedo-Escatel \& Dietrich, 2020a: 575

OSUC (holotype)

Endemic to Mexico (GRO)

\section{Genus Zabrosa Oman, 1949}

Zabrosa Oman, 1949: 128 (type species: Thamnotettix amazonensis Osborn, 1923)

Nearctic and Neotropical; distribution in Mexico Fig. 5U

Linnavuori (1959), Pinedo-Escatel \& Dietrich (2020b)

Fig. $8 \mathrm{U}$ and $\mathrm{Bb}$
Zabrosa amazonensis (Osborn, 1923)

Thamnotettix amazonensis Osborn, 1923: 65

Brazosa amazonensis: Oman, 1938: 386

Zabrosa amazonensis: Oman, 1949: 128

OSUC (holotype and paratypes); INHS, CAJAPE, and OSUC (other material)

Mexico (SLP; CHIS), USA, and Brazil

\section{DISCUSSION}

The tribe Athysanini is widely distributed in Mexico but individual species vary significantly in distribution and nearly two thirds of their diversity is concentrated in the Mexican Transition Zone of central Mexico, which is home to a diverse flora and fauna of Nearctic and Neotropical species (Figs 4 and 5, also see Fig. 6 in Pinedo-Escatel et al., 2021). Plant associations remain poorly known for most species and available data are mainly for groups residing within particular habitats, e.g., Tropical Dry Forest and Pine/Oak Forest (Aguilar-Pérez et al., 2019; PinedoEscatel et al., 2021). Despite extensive recent collecting, many species are still known only from the original type series collected in the 1930s and 40s and may be endangered or extinct (Pinedo-Escatel et al., 2021). Mexico harbors nearly $30 \%$ of the endemic genera of Athysanini recorded from the New World and harbors the largest number of endemic athysanine genera of any New World country (Oman et al., 1990; Pinedo-Escatel et al., 2021). Thus, additional collecting efforts, particularly in relatively intact tropical forests, will likely continue to yield new species of this group.

Identification of Neotropical genera of Athysanini, particularly those endemic to Mexico, is often difficult. DeLong's original descriptions and illustrations omit many important details and Linnavuori (1959) did not include most of the endemic Mexican genera described by DeLong in his key to Neotropical Deltocephalinae. Cwikla \& Blocker (1981) provided brief diagnoses and comparative notes for some of the endemic Mexican genera not included by Linnavuori (1959) but did not provide a revised key or additional illustrations. Thus, until now, identification of these genera has required review of original descriptions and illustrations in DeLong's numerous short taxonomic publications or reference to authoritatively identified specimens from DeLong's collection at Ohio State University.

The key to genera provided above represents a first step toward a more comprehensive revision of New World Athysanini. As noted previously, Athysanini is a poorly defined, polyphyletic group and its classification needs to be revised comprehensively based on phylogenetic analysis. The most comprehensive previous phylogenetic analysis of Deltocephalinae included only 16 endemic New World athysanine genera but recovered two almost exclusively New World clades comprising these endemic Athysanini and the endemic New World tribes Bahitini, Pendarini, and Scaphytopiini (Zahniser \& Dietrich, 2013). This suggests that the New World members of Athysanini evolved independently from Athysanini genera that are endemic to other continents or widespread in the Holarctic. More detailed phylogenetic analyses of these New World lineages 
are needed not only to elucidate the phylogenetic status of individual endemic tribes and genera but also to reveal patterns of biogeography, host and habitat use that could explain their evolutionary diversification.

ACKNOWLEDGMENTS. The first author is deeply grateful to the Centro Universitario de Ciencias Biológicas y Agropecuarias (CUCBA) of the Universidad de Guadalajara, Mexico and the Illinois Natural History Survey, University of Illinois at UrbanaChampaign, United States for their strong support during development of this research. The following individuals helped substantially with designing the project: D. Yassir, K. Missael, A Cristobal, J. Mario, M. Torres, B. Rodriguez, M. Jiménez. Fieldwork was done thank to: B. Valdez and J. Aguilar (CUCBA); J. Aragon, E. Limon, K. Machuca and P. Carrillo (Instituto de Botánica CUCBA); G. Rodríguez (Laboratorio de Entomología, Ecología de Insectos, CUCBA); D. Suarez, M. de Rocio and A. Trejo (Instituto Tecnológico de Conkal). Manuscript was improved by useful comments of: J. Romero and E. Blanco (Colegio de Postgradudados); B. Morris (INHS); D. Takiya (Federal University of Rio de Janeiro); S. McKamey (USNM); H. Brailovsky and C. Mayorga (CNIN). R. Torres, I. Mariana, and L. Valencia (CUCBA) reviewed an early draft. H. Fierros-Lopez (CUCBA) kindly drew Fig. 1B. Curators of the collections CZUG, OSUC, INHS, USNM, CNIN, and CEAM provided access to types and other reference material. The opinions expressed by individuals in this report do not necessarily represent the policies of the U.S. Department of Agriculture.

AUTHOR CONTRIBUTIONS. JAPE designed the study and wrote first draft of the manuscript; $\mathrm{CDH}$ wrote and edit manuscript; JNZ, GMR and LP reviewed and improved substantially the manuscript.

FUNDING. The first author thanks Consejo Nacional de Ciencia y Tecnología (CONACYT) for his PhD scholarship (number of cvu: 705854). This research was funded by the BEMARENA graduate department of CUCBA, the Rufford Foundation (grants: 25290-1 and 29982-2) and The Mohammed bin Zayed Species Conservation Fund (grant 180518408) to the first author. Second author was supported by the US National Science Foundation Grant DEB1639601.

\section{REFERENCES}

Aguilar-Pérez J.G., Pinedo-Escatel J.A. \& Valdez-Quezada B.C. 2019: Three new Mexican species of the endemic Athysanini leafhopper genus Devolana DeLong (Hemiptera: Cicadellidae) from the tropical dry forest. $-J$. Nat. Hist. 53: 2039-2056.

Anufriev G.A. \& Emeljanov A.F. 1988: Suborder Cicadinea (Auchenorrhyncha). In Lehr P.A. (ed.): Keys to the Insects of the Far East of the USSR. Homoptera and Hemiptera. Nauka, Moscow, pp. 1-496.

Ball E.D. 1901: New Jassidae from the Rocky Mountain and Pacific region. - Can. Entomol. 33: 4-11.

BALL E.D. 1907: The genus Eutettix, with especial reference to $E$. tenella, the Beef Leaf-Hopper: A taxonomic, biologic, and economic study of the North American forms. - Proc. Davenport Acad. Sci. 12: 27-94.

BALl E.D. 1911: Additions to the jassid fauna of North America (Homoptera). - Can. Entomol. 43:197-204.

BALL E.D. 1931: Some new genera and species of leafhoppers related to Mesamia Ball. - Bull. Brooklyn Entomol. Soc. 26: 91-95.
BALL E.D. 1936: Some new genera of leafhoppers related to Thamnotettix. - Bull. Brooklyn Entomol. Soc. 31: 57-60.

BALL E.D. 1937: Some new species of leafhoppers in groups recently segregated from Thamnotettix. - Bull. Brooklyn Entomol. Soc. 32: 26-31.

Beamer R.H. 1943: A new Atanus from Argentina, South America (Homoptera: Cicadellidae). — Proc. Entomol. Soc. Wash. 45: 178-179.

Blocker H.D. \& LARSEN K.J. 1991: A new leafhopper genus, Cocrassana (Homoptera: Cicadellidae), from Mexican Tripsacinae and a synopsis of related genera. - J. Kans. Entomol. Soc. 46: $123-126$.

CWikla P.S. \& Blocker H.D. 1991: Neotropical genera of Deltocephalinae not included in Linnavuori's $1959 \mathrm{key}$. - Bull. Entomol. Soc. Am. 27: 170-178.

DeLong D.M. 1943a: A new genus, Artucephalus, and a new species of Mexican leafhopper (Homoptera, Cicadellidae). Ann. Entomol. Soc. Am. 36: 654-655.

DeLong D.M. 1943b: A new genus (Deltorynchus) and two new species of leafhoppers (Homoptera: Cicadellidae) from Mexico. - Ohio J. Sci. 43: 79-80.

DeLong D.M. 1943c: A new genus Stoneana and three new species of Mexican leafhoppers (Homoptera, Cicadellidae). Ann. Entomol. Soc. Am. 36: 448-450.

DeLong D.M. 1944a: A new genus Mexicananus and species of leafhopper from Mexico related to Phlepsius and Texananus. -Ohio J. Sci. 44: 89.

DeLong D.M. 1944b: The genus Ollarianus (Homoptera: Cicadellidae) in North America, including Mexico. - J. Wash. Acad. Sci. 34: 391-298.

DeLong D.M. 1944c: A new genus Tenuisanus and species of Mexican leafhopper (Homoptera, Cicadellidae). - Bull. Brooklyn Entomol. Soc. 39: 73-74.

DeLong D.M. 1945a: A new genus Acunasus and eight new species of Mexican leafhoppers (Homoptera: Cicadellidae). Ann. Entomol. Soc. Am. 38: 199-206.

DeLong D.M. 1945b: The genus Chlorotettix (Homoptera: Cicadellidae) in Mexico. - Lloydia 8: 1-30.

DeLong D.M. 1945c: A new genus Retusanus and five new species of Mexican leafhoppers (Homoptera, Cicadellidae). Pan-Pac. Entomol. 21: 135-140.

DeLong D.M. 1946a: The Mexican species of Idiodonus (Homoptera: Cicadellidae). — Bull. Brooklyn Entomol. Soc. 46: 1330.

DeLong D.M. 1946b: A new genus (Costamia) and species of Mexican leafhopper (Homoptera: Cicadellidae). - Ann. Entomol. Soc. Am. 39: 82-83.

DeLong D.M. 1946c: A new genus (Excavanus) and species of Mexican leafhoppers related to Acunasus (Homoptera: Cicadellidae). - Ann. Entomol. Soc. Am. 39: 446-447.

DeLong D.M. 1947: A new genus Usanus and new species of Mexican leafhoppers related to Chlorotettix. - Pan-Pac. Entomol. 23: 110-111.

DeLong D.M. 1959: A new genus, Renonus, and two new species of Mexican leafhoppers (Homoptera: Cicadellidae). — Ohio J. Sci. 59: 325-326.

DeLong D.M. 1967a: Pseutettix, a new genus and two new species of Mexican Deltocephalinae. - Pan-Pac. Entomol. 43: 210-212.

DeLong D.M. 1967b: Spinulana, new genus of Mexican Deltocephalinae, and two new species of Spinulana (Homoptera: Cicadellidae). - Ohio J. Sci. 67: 20-22.

DeLong D.M. 1967c: Devolana, new genus of Mexican Deltocephalinae, and a new species of Devolana (Homoptera: Cicadellidae). - Ohio J. Sci. 67: 22-23. 
DeLong D.M. 1967d: Studies of the Mexican Deltocephalinae: a new genus, Conversana and three new species. - Proc. Entomol. Soc. Wash. 69: 266-269.

DeLong D.M. 1978: The genus Atanus (Homoptera: Cicadellidae) in North America. - J. Kans. Entomol. Soc. 51: 484-491.

DeLong D.M. 1980: New genera and species of Mexican and South American Deltocephalinae leafhoppers (Homoptera, Cicadellidae, Deltocephalinae). — Rev. Peruana Entomol. 23: 63-71.

DeLong D.M. 1983: New species and distribution notes of Mexican and Bolivian Idiodonus (Homoptera: Cicadellidae). - Entomol. News 94: 89-92.

DeLong D.M. 1984: A revised key to the Idiodonus (Homoptera: Cicadellidae) of Mexico and Bolivia. - Entomol. News 95: 9-15.

DeLong D.M. \& Caldwell J. S. 1937: Check List of the Cicadellidae (Homoptera) of North America. By the Authors, $95 \mathrm{pp}$.

DeLong D.M. \& Harlan H.J. 1968: Studies of the Mexican Deltocephalinae: New species of Eutettix and two allied new genera (Homoptera: Cicadellidae). — Ohio J. Sci. 68: 139-152.

DeLong D.M. \& Hershberger R.V. 1947a: A new genus and species of leafhopper from Central America related to Atanus (Homoptera: Cicadellidae). — Proc. Entomol. Soc. Wash. 84: 231-232.

DeLong D.M. \& Hershberger R.V. 1947b: A new genus (Crassana), new subgenus (Macrasana) and new species of North American leafhoppers (Homoptera: Cicadellidae). - PanPac. Entomol. 23: 76-78.

DeLong D.M. \& Hershberger R.V. 1947c: The genus Mesamia in Mexico (Homoptera: Cicadellidae). - Ann. Entomol. Soc. Am. 40: 257-265.

DeLong D.M. \& Hershberger R.V. 1948a: A new genus, Dampfiana, and new species of leafhoppers related to Stoneana (Homoptera: Cicadellidae). — Proc. Entomol. Soc. Wash. 50: 229-230.

DeLong D.M. \& Hershberger R.V. 1948b: A new genus, Neodo$n u s$, and species of Mexican leafhopper (Homoptera, Cicadellidae) related to Idiodonus. - Bull. Brooklyn Entomol. Soc. 42: $159-161$.

Dietrich C.H. 2005: Keys to the families of Cicadomorpha and subfamilies and tribes of Cicadellidae (Hemiptera: Auchenorrhyncha). - Fla Entomol. 88: 502-517.

HePNER L.W. 1942a: A revision of the genus Aligia (Homoptera, Cicadellidae) north of Mexico. - Univ. Kans. Sci. Bull. 28: 145-183.

HepNER L.W. 1942b: Taxonomic revision of the genus Eutettix in America north of Mexico (Homoptera, Cicadellidae). - Univ. Kans. Sci. Bull. 28: 253-293.

KNULl D.J. 1946: The Genus Bandara Ball (Homoptera: Cicadellidae). — Ann. Entomol. Soc. Am. 39: 259-264.

Kramer J.P. \& DeLong D.M. 1968: Studies of the Mexican Deltocephalinae: Aligia and some new allied genera and species (Homoptera: Cicadellidae). — Ohio J. Sci. 68: 169-175.

Kramer J.P. \& DeLong D.M. 1969: Studies of the Mexican Deltocephalinae II. Seven new species of Norvellina (Homoptera: Cicadellidae). - Ohio J. Sci. 69: 115-121.

Lindsay D.R. 1939: The genus Norvellina. - Univ. Kans. Sci. Bull. 26: 169-213.

LINNAVUORI R. 1959: Revision of the Neotropical Deltocephalinae and some related subfamilies (Homoptera). - Ann. Zool. Soc. Zool. Bot. Fenn. 'Vanamo' 20: 1-370.

LinNAVUORI R. \& DeLong D.M. 1978: Seventeen new species and three new genera of Central and South American Deltocephalini (Homoptera: Cicadellidae). — Brenesia 14/15: 195-226.
Morrone J.J., Escalante T. \& Rodriguez-Tapia G. 2017: Mexican biogeographic provinces: Map and shapefiles. - Zootaxa 4277: 277-279.

Nielson M.W. 1957: A revision of the genus Colladonus (Homoptera, Cicadellidae). - USDA Tech. Bull. No. 1156, 52 pp., 13 pls.

Nielson M.W. 1988: Colladonus and related genera of Mexico and Central America with new taxa and synonymy (Homoptera: Cicadellidae). — Great Basin Nat. 12: 103-134.

OMAN P.W. 1938: A generic revision of American Bythoscopinae and South American Jassinae. - Univ. Kans. Sci. Bull. 24: 343-420.

OMAN P.W. 1949: The Nearctic leafhoppers (Homoptera: Cicadellidae). A generic classification and check list. - Mem. Entomol. Soc. Wash. 3: 1-253.

Oman P.W., Knight W.J. \& Nielson M.W. 1990: Leafhoppers (Cicadellidae): A Bibliography, Generic Check-list and Index to the World Literature 1956-1985. CAB International, Wallingford, $365 \mathrm{pp}$.

Pinedo-Escatel J.A. \& Dietrich C.H. 2020a: Nomenclatural changes and two new species in the leafhopper genus Usanus DeLong (Hemiptera: Cicadellidae) with notes on conservation status. - Zootaxa 4822: 567-576.

Pinedo-Escatel J.A. \& Dietrich C.H. 2020b: Review of the enigmatic Neotropical leafhopper genus Brazosa Oman and other potentially related Athysanini genera (Hemiptera: Auchenorrhyncha: Cicadellidae: Deltocephalinae), with descriptions of South American new genera and species. - Zootaxa 4830: 401-454.

Pinedo-Escatel J.A., Zahniser J.N., Dietrich C.H. \& Moya-RayGOZA G. 2016: Duocrassana longula, new genus and species of leafhopper (Hemiptera: Cicadellidae: Deltocephalinae) from southern Mexico and its relationship to other genera in Athysanini. - Zootaxa 4196: 579-588.

Pinedo-Escatel J.A., Dietrich C.H., Mckamey S.H. \& MoyaRAYGOZA G. 2018: Nomenclatural changes in the grass-feeding Mexican leafhopper genus Cocrassana Blocker \& Larsen (Hemiptera: Cicadellidae: Deltocephalinae: Athysanini). - Rev. Bras. Entomol. 62: 176-179.

Pinedo-Escatel J.A., Aragón-Parada J., Dietrich C.H., MoyaRaygoza G., Zahniser J.N. \& Portillo L. 2021: Biogeographical evaluation and conservation assessment of arboreal leafhoppers in the Mexican Transition Zone biodiversity hotspot. - Diversity Distrib. In Press.

RaKitov R.A. 1998: On differentiation of cicadellid leg chaetotaxy (Homoptera: Auchenorrhyncha: Membracoidea). - Russ. Entomol. J. 6: 7-27.

Ribaut H. 1942: Demembrement des generes Athysanus Burm. et Thamnotettix Zett. (Homoptera-Jassidae). - Bull. Soc. Hist. Nat. Toulouse 77: 259-270.

STÅL C. 1864: Hemiptera mexicana enumeravit speciesque novas descripsit. - Entomol. Ztg. (Stettin) 25: 49-86.

VAN DUZEe E.P. 1892: A synoptical arrangement of the genera of the North American Jassidae, with descriptions of some new species. - Trans. Am. Entomol. Soc. 19: 295-307.

VAN Duzee E.P. 1894: A catalogue of the described Jassoidea of North America. - Trans. Am. Entomol. Soc. 21: 245-317.

VAN DuZEe E.P. 1916: Suborder Homoptera Latr. 1810. Section Auchenorhyncha A. \& S. 1843. In: Check list of Hemiptera (excepting the Aphididae, Aleurodidae and Coccidae) of America North of Mexico. New York Entomological Society, New York, $\mathrm{xi}+111 \mathrm{pp}$.

Zahniser J.N. \& Dietrich C.H. 2013: A review of the tribes of Deltocephalinae (Hemiptera: Auchenorrhyncha: Cicadellidae). - Eur. J. Taxon. 45: 1-211. 
Zahniser J.N., McKamey S.H. \& Dmitriev D.A. 2012: Nomenclatural changes and notes in the Deltocephalinae (Hemiptera: Cicadellidae). — Pan-Pac. Entomol. 88: 356-364.

Zanol K.M.R. 1989: Nova especie de Carelmapu Linnavuori, 1959 (Homoptera: Cicadellidae). — An. Soc. Entomol. Bras. 18: $367-369$.
ZANOL K.M.R. 2008: Catalogue of the Neotropical Deltocephalinae (Hemiptera: Cicadellidae). Part III - Tribe Athysanini. Acta Biol. Parana. 36: 1-46.

Received February 2, 2021; revised and accepted July 23, 2021 Published online September 17, 2021

Online supplementary file:

Table S1 (http://www.eje.cz/2021/027/S01.pdf). 